\title{
The OH-streamer in Sagittarius A revisited: analysis of hydroxyl absorption within 10 pc from the Galactic centre ${ }^{\star, \star \star, \star \star \star}$
}

\author{
R. Karlsson ${ }^{1}$, Aa. Sandqvist ${ }^{1}$, K. Fathi ${ }^{1,2}$, and S. Martín ${ }^{3}$ \\ ${ }^{1}$ Stockholm Observatory, Stockholm University, AlbaNova University Center, 10691 Stockholm, Sweden \\ e-mail: rolandk@astro.su.se \\ 2 Oskar Klein Centre for Cosmoparticle Physics, Stockholm University, 10691 Stockholm, Sweden \\ ${ }^{3}$ Institut de RadioAstronomie Millimétrique 300 rue de la Piscine, Domaine Universitaire 38406 Saint-Martin d'Hères, France
}

Received 18 June 2014 / Accepted 23 June 2015

\begin{abstract}
Aims. We study the structure and kinematics of the $\mathrm{OH}$-streamer and the $+80 \mathrm{~km} \mathrm{~s}^{-1}$ cloud and their interactions with the circumnuclear disk (CND) and with other molecular clouds in the vicinity of the Galactic centre (GC), and we map OH absorption at about 6" resolution at $R \leq 10 \mathrm{pc}$ from the $\mathrm{GC}$, with about $9 \mathrm{~km} \mathrm{~s}^{-1}$ of velocity resolution.

Methods. The VLA was used to map OH line absorption at the 1665 and $1667 \mathrm{MHz}$ lambda doublet main lines of the ${ }^{2} \Pi_{3 / 2}$ state towards the Sagittarius A complex.

Results. Strong OH absorption was found in the OH-streamer, the southern streamer (SS), the $+20,+50$, and $+80 \mathrm{~km} \mathrm{~s}^{-1} \mathrm{molecular}$ clouds, the molecular belt, the CND, the expanding molecular ring (EMR), and the high negative velocity gas (HNVG). The OHstreamer was found to comprise three parts, head, mid, and tail, and to interact with the SS/ $+20,+80 \mathrm{~km} \mathrm{~s}^{-1}$ clouds and the CND. Optical depths and column densities divided by excitation temperatures have been calculated for the OH-streamer and the $+80 \mathrm{~km} \mathrm{~s}^{-1}$ cloud.

Conclusions. The $\mathrm{OH}$-streamer, the SS, the +20 and $+80 \mathrm{~km} \mathrm{~s}^{-1}$ clouds, and the CND are intimately related in position and velocity space. The $\mathrm{OH}$-streamer was found to be a clumpy object stretching in projection from the inner radius of the CND at about $1.8 \mathrm{pc}$ from Sgr A*towards and partly engulfing Sgr A*. As a side result of our data, a possible link between the near side of the EMR and the CND's southwest lobe was found. Additionally, we found $\mathrm{OH}$ absorption against all four of the previously known compact H II regions A-D, located east of Sgr A East, indicating their close association with the $+50 \mathrm{~km} \mathrm{~s}^{-1}$ cloud.
\end{abstract}

Key words. Galaxy: center - ISM: individual objects: Sagittarius A - ISM: molecules - ISM: clouds

\section{Introduction}

At the very core of the Milky Way Galaxy is the $\sim 4$-millionsolar-mass supermassive black hole (SMBH) whose non-thermal radio continuum signature is called $\mathrm{Sgr}^{*}$. Surrounding it at a distance of one to a few pc and orbiting it with a velocity of about $100 \mathrm{~km} \mathrm{~s}^{-1}$ is a rotating molecular structure called the circumnuclear disk (CND). In the cavity inside the CND, there is a central cluster of old stars and bright young stars clustered around the $\mathrm{SMBH}$. Inside the cavity is also the mini-spiral-shaped $\mathrm{H}$ II region called Sgr A West, which represents the inner western edge of the CND, and a northern streamer extending from the CND towards Sgr A*. A little farther out there is a large molecular belt extending from the southwest to the northeast (parallel to the plane of the Galaxy). The major components of this belt are the two giant molecular clouds, known as the $+20 \mathrm{~km} \mathrm{~s}^{-1}$ cloud and the $+50 \mathrm{~km} \mathrm{~s}^{-1}$ cloud. A giant energetic $\left(>10^{52} \mathrm{erg}\right)$ supernovaremnant-like non-thermal continuum radio shell (diameter about $8 \mathrm{pc}$ ), known as Sgr A East, is plowing into this molecular belt

\footnotetext{
* Based on observations with the Karl G. Jansky Very Large Array, NRAO.

$\star \star$ The 1665 and $1667 \mathrm{MHz}$ data cubes, presented in

Appendices B-E, respectively, are only available at the CDS via anonymous ftp to cdsarc.u-strasbg. fr (130.79.128.5) or via http://cdsarc.u-strasbg.fr/viz-bin/qcat?J/A+A/582/A118 $\star \star \star$ Appendices are available in electronic form at http://www . aanda.org
}

from the side near $\mathrm{Sgr}^{*}$, creating regions of shock interaction especially at the inner surface of the $+50 \mathrm{~km} \mathrm{~s}^{-1}$ cloud. Lunar occultation observations of $\mathrm{OH}$ absorptions at $\sim 40^{\prime \prime}$ resolution showed the multiple structures within the Sgr A region (Kerr \& Sandqvist 1968; Sandqvist 1973, 1974). Interferometric observations of $\mathrm{OH}$ absorption in the Sgr A complex with an angular resolution of 4.5 were subsequently presented by Bieging (1976). Absorption measurements are useful for revealing the location of the clouds along the line of sight, relative to the continuum emission.

General reviews of the Galactic centre (GC) region have been presented by Mezger et al. (1996) and Morris \& Serabyn (1996), among others, and an up-to-date introduction to the Sgr A complex is given by Ferrière (2012).

We performed observations of the GC region in all four $18-\mathrm{cm} \mathrm{OH}$ lines with the Karl G. Jansky Very Large Array, henceforth the VLA, in the wide array BnA configuration and in the two main $\mathrm{OH}$ lines also in the compact DnC configuration. The first preliminary $1667 \mathrm{MHz} \mathrm{OH}$ line results with about 4" angular resolution were presented by Sandqvist et al. (1987, 1989). One of the most interesting results was the discovery of an "OH-streamer" that stretches from the southwest part of the CND and subsequently sweeps northeastward projected inside the CND towards Sgr A*. However, the velocities of the $\mathrm{OH}$-streamer and the SW CND differed by more than $100 \mathrm{~km} \mathrm{~s}^{-1}$ so their relation was unclear. Subsequently, 
the OH-streamer was also detected at 1612 and $1665 \mathrm{MHz}$ in the high-resolution BnA observations with the VLA (Karlsson et al. 2003). The full extent of the $\mathrm{OH}$-streamer has not been detected in other molecular lines, probably owing to a lack of angular resolution and sensitivity. However, recent interferometric observations of $\mathrm{CN} J=2-1$ emission by Martín et al. (2012) have revealed a few high-density clumps in the $\mathrm{OH}$-streamer. No magnetic field with a $3 \sigma$ upper limit of $2 \mathrm{mG}$ has been found in the OH-streamer (Killeen et al. 1992). Sandqvist et al. (1987) also briefly noted "another feature at $+78 \mathrm{~km} \mathrm{~s}^{-1}$ " (henceforth the $+80 \mathrm{~km} \mathrm{~s}^{-1}$ cloud) that seemed related to the OH-streamer.

In this paper, we present the concatenated VLA BnA and DnC observations of the two 1665 and $1667 \mathrm{MHz}$ main $\mathrm{OH}$ lines. We concentrate here on the interplay between the $\mathrm{OH}-$ streamer, the $+80 \mathrm{~km} \mathrm{~s}^{-1}$ cloud, and the CND and possible effects of the SMBH and the surrounding star cluster. Moreover, a possbile link between the CND SW lobe and the near side of the expanding molecular ring (EMR) is suggested. A detailed study of all the features seen in the data and their interrelations is, however, beyond the present scope and will be the subject of a subsequent paper.

\section{Observations}

The main $\mathrm{OH}$ transition lines at 1665 and $1667 \mathrm{MHz}$ and the satellite lines at 1612 and $1720 \mathrm{MHz}$ were observed in June 1986 with the VLA in the BnA wide array configuration. The main transition lines were also observed with the VLA in its DnC compact array configuration in October 1989. The initial- and post-calibration of the observational data were done with the National Radio Astronomy Observatory (NRAO), Astronomical Image Processing System (AIPS) at the VLA site and at the Array Operations Center in Socorro, NM. The two sets of $(u, v)$ data for each of the main lines were subsequently concatenated into one data set to improve sensitivity and reduce the effects of missing zero-spacing observations. The concatenation was done with the NRAO AIPS DBCON program package, and baselines were subtracted with the NRAO AIPS UVLSF program package in the $(u, v)$-plane. Throughout this paper we define "OH absorption" as "continuum minus line flux" $\left(=-T_{\mathrm{L}}\right)$, in units of $\mathrm{mJy} /$ beam. The angular resolution is $7^{\prime \prime} \times 5^{\prime \prime}$, and the velocity resolution is $8.8 \mathrm{~km} \mathrm{~s}^{-1}$. Typical noise levels in the concatenated spectral line maps and line profiles are about $25 \mathrm{mJy} / \mathrm{beam}$, and about $5 \mathrm{mJy} /$ beam for the original $4{ }^{\prime \prime} .0 \times 22^{\prime \prime} 8$ angular resolution BnA $1667 \mathrm{MHz}$ data. The concatenated data are used for the main part of this paper, but the original BnA $1667 \mathrm{MHz}$ data are used as a complement. The subsequent processing of the data was done with both the NRAO AIPS and the $\mathrm{XS}^{1}$ program packages. Projected distances correspond to about $0.04 \mathrm{pc} /{ }^{\prime \prime}$ assuming a distance to the GC of $8 \mathrm{kpc}$. The position of Sgr A* is $(\mathrm{RA}, \mathrm{Dec})=17^{\mathrm{h}} 45^{\mathrm{m}} 40^{\mathrm{s}} .03,-29^{\circ} 00^{\prime} 27^{\prime \prime} \cdot 6$ (J2000.0). For further information about the observations, see Karlsson et al. (2003, 2013).

\section{Results}

\subsection{Morphology and physical properties of the $\mathrm{OH}$-streamer and the $+80 \mathrm{~km} \mathrm{~s}^{-1}$ cloud}

The OH-streamer is observed at $1667 \mathrm{MHz}$ between velocities of -29 and $67 \mathrm{~km} \mathrm{~s}^{-1}$ (Figs. 1 and 2; and Figs. B.1-B.3, C.1-C.3). At $1665 \mathrm{MHz}$ it is observed between -20 and $68 \mathrm{~km} \mathrm{~s}^{-1}$

1 Developed by P. Bergman, Onsala Space Observatory.

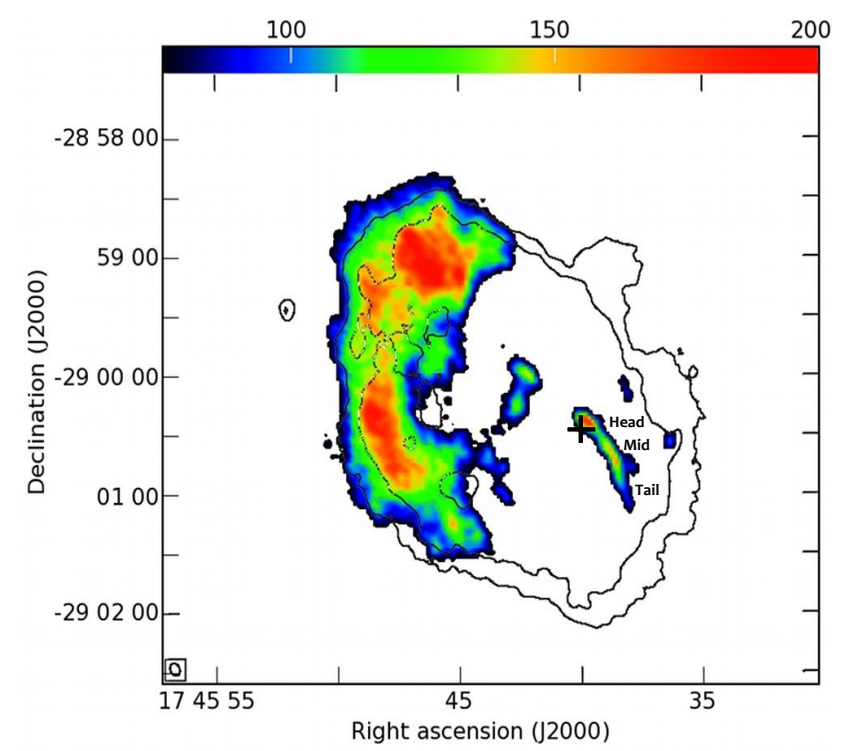

Fig. 1. $\mathrm{OH}$ absorption, continuum minus line flux, at $1667 \mathrm{MHz}$ shown in colour shades at a velocity of $59 \mathrm{~km} \mathrm{~s}^{-1}$. The contour lines depict the $18 \mathrm{~cm}$ continuum emission of the Sgr A complex at intensities of 100 and $150 \mathrm{mJy} /$ beam. Sgr $\mathrm{A}^{*}$ is indicated with a plus sign. The $\mathrm{OH}-$ streamer is seen running in a northeasterly direction and ends slightly northwest of the position of Sgr A*, and its parts are labelled in the figure. The $+50 \mathrm{~km} \mathrm{~s}^{-1}$ cloud is seen east of Sgr A*

(Figs. D.1 and E.1). It is slightly curved to the E with an average position angle (PA) of $\sim 28^{\circ}$ east of the north celestial pole. The projected length of the $\mathrm{OH}$-streamer is $\sim 68^{\prime \prime}(2.7 \mathrm{pc})$, and the width is $\sim(4-15)^{\prime \prime}(0.2-0.6 \mathrm{pc})$, as measured at $59 \mathrm{~km} \mathrm{~s}^{-1}$. Three clumps, head, mid, and tail, are seen inside of the $\mathrm{OH}$ streamer at velocities of 50,59, and $67 \mathrm{~km} \mathrm{~s}^{-1}$ in both the 1665 and $1667 \mathrm{MHz}$ transitions. Inside of the mid part, a fine structure of smaller clumps is also observed.

The geometry of $\mathrm{OH}$-absorption in the head varies significantly with velocity, as can be seen in Fig. 3, where we present the higher resolution BnA maps from Karlsson et al. (2003). A winglike structure develops on the western side at velocities of about $50 \mathrm{~km} \mathrm{~s}^{-1}$ and displays an anti-clockwise rotation as the velocity decreases, and the head becomes nearly circular at velocities below $15 \mathrm{~km} \mathrm{~s}^{-1}$.

The $+80 \mathrm{~km} \mathrm{~s}^{-1}$ cloud is observed at a distance of about $18^{\prime \prime}$ $(0.7 \mathrm{pc}) \mathrm{W}$ of $\mathrm{Sgr} \mathrm{A}^{*}$ at velocities between 67 and $111 \mathrm{~km} \mathrm{~s}^{-1}$ (Figs. 2, 4 and B.1, C.1). It is extended mainly in the north south direction. The length extends to $65^{\prime \prime}(2.6 \mathrm{pc})$, while the width is about $15^{\prime \prime}(0.6 \mathrm{pc})$ at $76 \mathrm{~km} \mathrm{~s}^{-1}$. Two clumps are seen in the $+80 \mathrm{~km} \mathrm{~s}^{-1}$ cloud between $76 \mathrm{~km} \mathrm{~s}^{-1}$ and $103 \mathrm{~km} \mathrm{~s}^{-1}$, separated by $\sim 25^{\prime \prime}(1 \mathrm{pc})$. At 103 and $111 \mathrm{~km} \mathrm{~s}^{-1}$, the northern part of the cloud bends to the east, and points towards the northeastern extension of the CND northeastern lobe in a similar manner to a $\mathrm{CN}$ emitting feature, centred at $90 \mathrm{~km} \mathrm{~s}^{-1}$ in a $30 \mathrm{~km} \mathrm{~s}^{-1}$ wide bin (Martín et al. 2012).

By dividing the spectral line maps $\left(-T_{\mathrm{L}}\right)$ by the continuum map $\left(T_{\mathrm{C}}\right)$, we reduce the effects of the varying continuum intensity in the region and obtain $\left(-T_{\mathrm{L}} / T_{\mathrm{C}}\right)$ maps at $1667 \mathrm{MHz}$ and $1665 \mathrm{MHz}$. This ratio is proportional to the optical depth as $-T_{\mathrm{L}} / T_{\mathrm{C}}=1-\mathrm{e}^{-\tau}$, where $1-\mathrm{e}^{-\tau}$ is often referred to as "apparent opacity". Both the $\mathrm{OH}$-streamer and the $+80 \mathrm{~km} \mathrm{~s}^{-1}$ cloud retain their basic properties in Figs. C. 1 and C.2, which validates the reality of the objects.

$\mathrm{OH}$ absorption line profiles were produced at 16 positions centred along the $\mathrm{OH}$-streamer and the $+80 \mathrm{~km} \mathrm{~s}^{-1}$ cloud, 


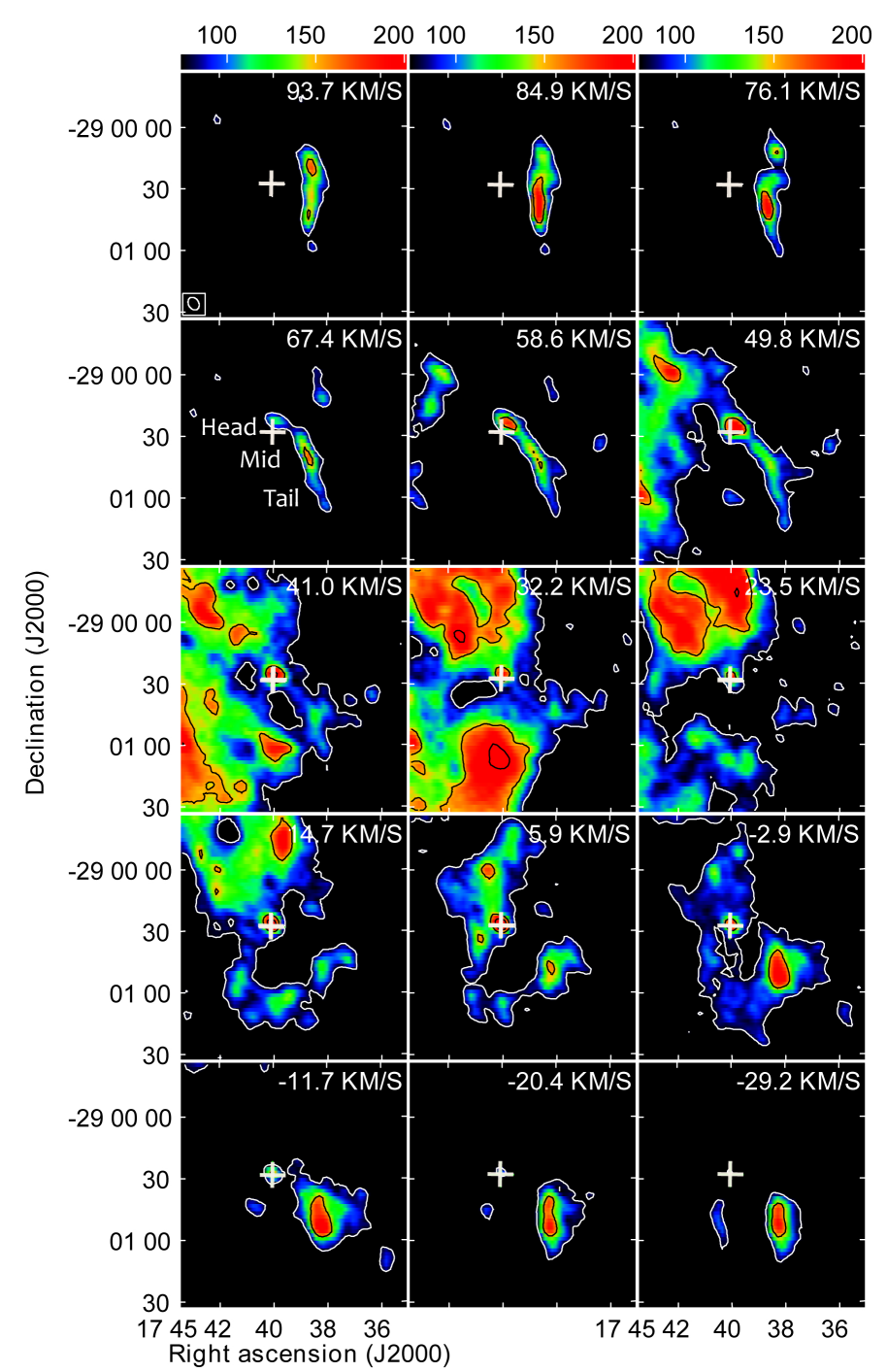

Fig. 2. OH absorption at $1667 \mathrm{MHz}$ towards the inner $\sim 110^{\prime \prime}(\sim 4.4 \mathrm{pc})$ of the GC at velocities between -29 and $94 \mathrm{~km} \mathrm{~s}^{-1}$. The lowest contour level is $75 \mathrm{mJy} / \mathrm{beam}, \sim 3 \sigma$, and the contour spacing is $\sim 3 \sigma$. The position of Sgr A* is marked with a plus sign. The $\mathrm{OH}$-streamer is seen at velocities between -29 and $67 \mathrm{~km} \mathrm{~s}^{-1}$, the $+80 \mathrm{~km} \mathrm{~s}^{-1}$ cloud at velocities from 76 to $94 \mathrm{~km} \mathrm{~s}^{-1}$, and the CND SW lobe between -29 and $15 \mathrm{~km} \mathrm{~s}^{-1}$. The three parts of the $\mathrm{OH}$-streamer are labelled in the $67 \mathrm{~km} \mathrm{~s}^{-1}$ panel. The SS and the $+20 \mathrm{~km} \mathrm{~s}^{-1}$ cloud can be seen south of Sgr A* in the 24 to $41 \mathrm{~km} \mathrm{~s}^{-1}$ panels, and are most prominent at $32 \mathrm{~km} \mathrm{~s}^{-1}$. The beam size is shown in the lower left corner of the uppermost left panel.

respectively (Figs. A.1 and A.2). Optical depths $\left(\tau_{1667}\right)$ and column densities divided by excitation temperatures $\left(N(\mathrm{OH}) / T_{\mathrm{ex}}\right)$ were calculated at the positions of the profiles as described in Karlsson et al. (2013; see Table A.1).

To get a first order of the mass of the $\mathrm{OH}$-streamer and the $+80 \mathrm{~km} \mathrm{~s}^{-1}$ cloud, we assume that each of the three clumps in the $\mathrm{OH}$-streamer and the full length of the $+80 \mathrm{~km} \mathrm{~s}^{-1}$ cloud can be represented by prolate ellipsoids (see Table 1). The material between the clumps in the $\mathrm{OH}$-streamer are not considered, such that the mass of the $\mathrm{OH}$-streamer is considered as a lower limit. The value of $n_{\mathrm{H}_{2}}$ is taken as $10^{5} \mathrm{~cm}^{-3}$, which is in the lower range for molecular gas in the CND found in Ferrière (2012). We derive at a total mass of $\gtrsim 400 M_{\odot}$ for the $\mathrm{OH}$-streamer and about $2500 M_{\odot}$ for the more massive $+80 \mathrm{~km} \mathrm{~s}^{-1}$ cloud (Table 1).
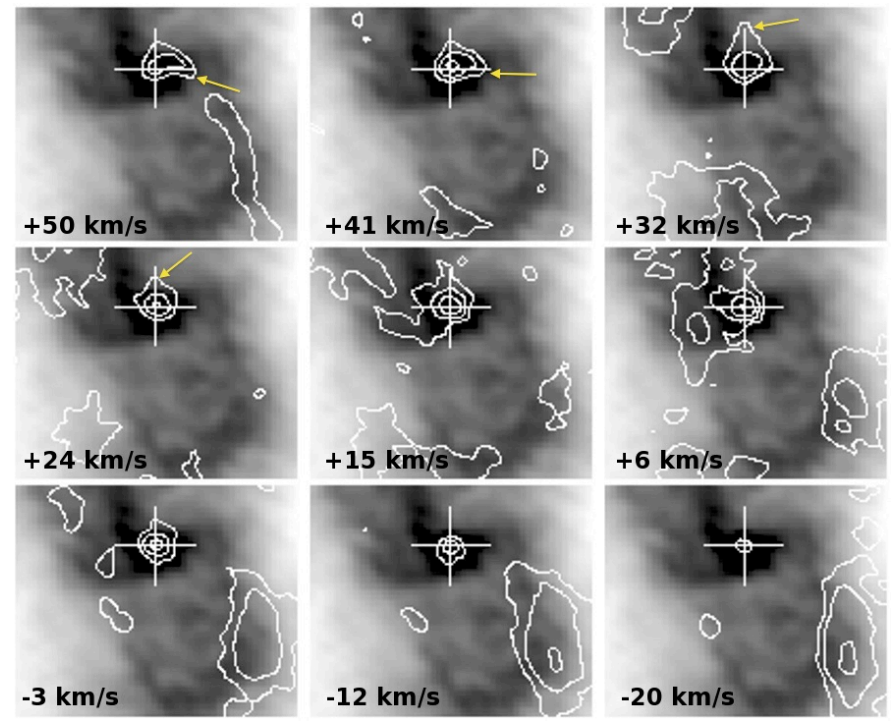

Fig. 3. OH absorption of the $\mathrm{OH}$-streamer head at $1667 \mathrm{MHz}$, observed with the VLA in BnA configuration at an angular resolution of $4.0^{\prime \prime} \times$ 2.8" (Karlsson et al. 2003) overlaid on the $18 \mathrm{~cm}$ continuum. We note the location of the central parts of Sgr A West in dark shades of grey. Sgr A* is labelled with a plus sign, and each panel covers a region of about $45^{\prime \prime} \times 45^{\prime \prime}$. The lowest contour level corresponds to about $4 \sigma$, and the velocity is given in the left-hand lower corner in each panel. Note the winglike appearance of the head, marked by an arrow, and its anti-clockwise rotation as the velocity decreases from 50 to $24 \mathrm{~km} \mathrm{~s}^{-1}$.

Table 1. Properties of the $\mathrm{OH}$-streamer and the $+80 \mathrm{~km} \mathrm{~s}^{-1}$ cloud (assuming $n_{\mathrm{H}_{2}}=10^{5} \mathrm{~cm}^{-3}$ ).

\begin{tabular}{lcccc}
\hline \hline Object & $\begin{array}{c}\text { Length } \times \text { Width } \\
\left({ }^{\prime \prime} \times{ }^{\prime \prime}\right)(\mathrm{pc} \times \mathrm{pc})\end{array}$ & $\begin{array}{c}D^{a} \\
(\mathrm{pc})\end{array}$ & $\begin{array}{c}\text { Volume } \\
\left(\mathrm{pc}^{3}\right)\end{array}$ & $\begin{array}{c}\text { Mass } \\
\left(M_{\odot}\right)\end{array}$ \\
\hline Head & $(15 \times 5)(0.6 \times 0.2)$ & 0.3 & 0.013 & 65 \\
Mid & $(20 \times 5)(0.8 \times 0.2)$ & 0.9 & 0.017 & 85 \\
Tail & $(15 \times 10)(0.6 \times 0.4)$ & 1.5 & 0.050 & 250 \\
$+80 \mathrm{~km} \mathrm{~s}^{-1} \mathrm{cl}$. & $(65 \times 15)(2.6 \times 0.6)$ & 0.7 & 0.490 & 2450 \\
\hline
\end{tabular}

Notes. ${ }^{(a)}$ Projected distance from the position of Sgr A*.

\subsection{Locations and interactions of the components}

We present here three different displays of our data to shed some light on possible links between the objects: i) maps of projected locations; ii) position-velocity diagrams; and iii) a position angle-velocity diagram.

i) Projected locations: to study the relative positions of the $\mathrm{OH}$-streamer, the $+80 \mathrm{~km} \mathrm{~s}^{-1}$ cloud, and the CND southwest lobe, we have overlaid those three components in Fig. 4. The $\mathrm{OH}$-streamer head is observed in absorption against Sgr A*, symmetrically between velocities of -29 to $15 \mathrm{~km} \mathrm{~s}^{-1}$. At 24 to $67 \mathrm{~km} \mathrm{~s}^{-1}$, the absorption moves to the northwest and is then only partly seen against Sgr A* (Figs. 2-4). The mid part overlaps the southern part of the $+80 \mathrm{~km} \mathrm{~s}^{-1}$ cloud (Fig. 4). The tail partially overlaps the CND southwestern lobe in Fig. 4. In Fig. 2 the northern part of the $+20 \mathrm{~km} \mathrm{~s}^{-1}$ cloud and the SS are clearly seen about $60^{\prime \prime}(2.4 \mathrm{pc})$ south of the position of Sgr A*, at $32 \mathrm{~km} \mathrm{~s}^{-1}$, which also is in the region of the southwest lobe of the CND.

Three blobs of CN $J=2-1$ emission (Martín et al. 2012) are observed in the $\mathrm{OH}$-streamer head, mid, and tail (Fig. 5). Moreover, $\mathrm{C}^{34} \mathrm{~S}$ emission is also found in the head, as seen 


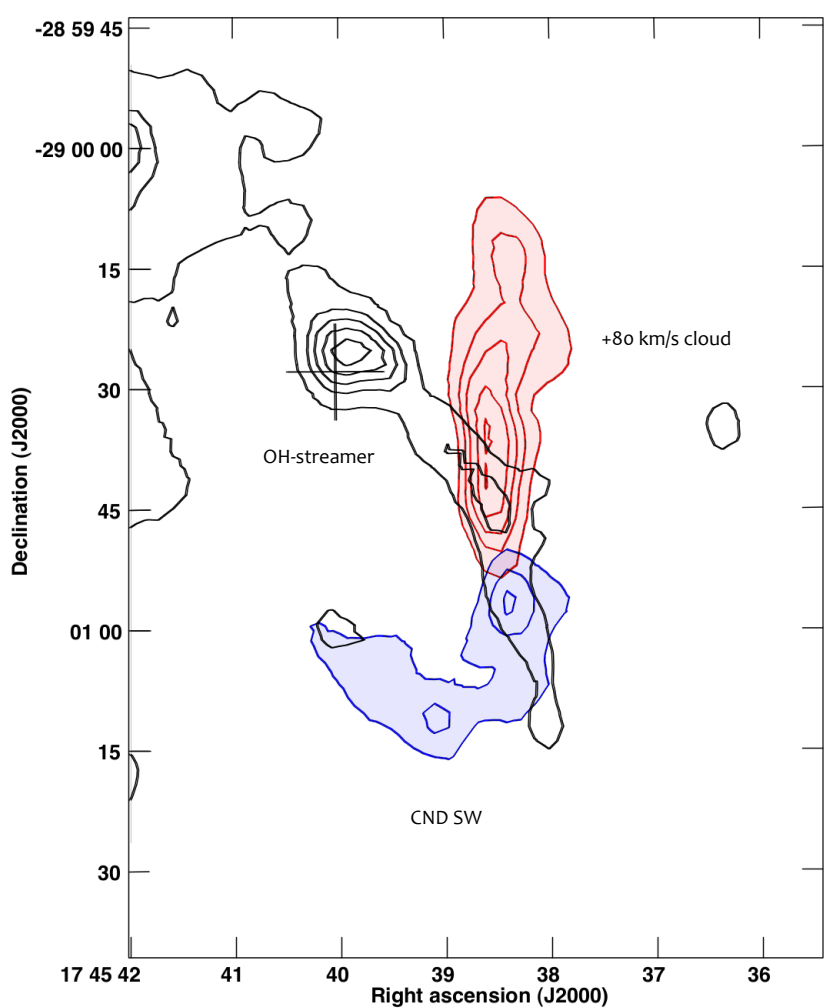

Fig. 4. $\mathrm{OH}$ absorption at $1667 \mathrm{MHz}$ of the $\mathrm{OH}$-streamer at $50 \mathrm{~km} \mathrm{~s}^{-1}$ with overlays of the $+80 \mathrm{~km} \mathrm{~s}^{-1}$ cloud at $85 \mathrm{~km} \mathrm{~s}^{-1}$, and the SW lobe of the CND at $-73 \mathrm{~km} \mathrm{~s}^{-1}$. The lowest contour level is drawn at $\sim 90 \mathrm{mJy} /$ beam $(\sim 3.5 \sigma)$ and the contour spacing is $1 \sigma$. Sgr A* is marked with a plus sign.

in the lower left-hand panel of Fig. 6 in Liu et al. (2012). Both the $\mathrm{CN} J=2-1$ and $\mathrm{C}^{34} \mathrm{~S} J=7-6$ emission trace high-density regions.

ii) Position-velocity diagrams: Position-velocity cuts of $\mathrm{OH}$ absorption at $1667 \mathrm{MHz}$ for a $2^{\prime} \times 2^{\prime}$ region around $\mathrm{Sgr} \mathrm{A}^{*}$ are shown in Fig. 6 and provide a detailed picture of interactions in the position-velocity space. Figure 6e is the RA scan at the declination of $\mathrm{Sgr} \mathrm{A}^{*}$, and it passes from the $+80 \mathrm{~km} \mathrm{~s}^{-1}$ cloud to the head of the $\mathrm{OH}$-streamer. In the head, both negative and positive velocities appear. In Fig. 6d, the $+80 \mathrm{~km} \mathrm{~s}^{-1}$ cloud displays an increasing velocity from about 63 to $107 \mathrm{~km} \mathrm{~s}^{-1}$ in the easterly direction towards the CND NE extension, corresponding to a velocity gradient of about $4.9 \mathrm{~km} \mathrm{~s}^{-1} /{ }^{\prime \prime}$. A rapid acceleration, from about 30 to $80 \mathrm{~km} \mathrm{~s}^{-1}$ is also observed in the western part of the molecular belt/ $+20 \mathrm{~km} \mathrm{~s}^{-1}$ cloud, seen as the knee-like structure at an (RA) of about $17^{\mathrm{h}} 45^{\mathrm{m}} 38^{\mathrm{s}} .5$ in Figs. 6f and g. This occurs in the overlap region of the $\mathrm{OH}$-streamer mid and the southern part of the $+80 \mathrm{~km} \mathrm{~s}^{-1}$ cloud. Moreover, the $\Delta V_{\text {FWHM }}$ increases from 26 to $50 \mathrm{~km} \mathrm{~s}^{-1}$ in this region.

Figure 7 is a visualisation throughout the entire data cube, covering an $\sim 7^{\prime} \times 7^{\prime}$ region seen face-on from the declination side. It illustrates that right ascension structure can also be easily followed at the same time. The corresponding visualisation seen from the right ascension-velocity side is shown in Fig. A.3. The OH-streamer tail is seen to interact with the $+20 \mathrm{~km} \mathrm{~s}^{-1}$ cloud, and interactions between the $+20,+80 \mathrm{~km} \mathrm{~s}^{-1}$ clouds can also be traced in Fig. 7. In those two figures the near side of the EMR seems to connect to the CND SW lobe. The interaction occurs at (RA, Dec) $=17^{\mathrm{h}} 45^{\mathrm{m}} 39^{\mathrm{s}},-29^{\circ} 00^{\prime} 55^{\mathrm{m}}$, and the map position of

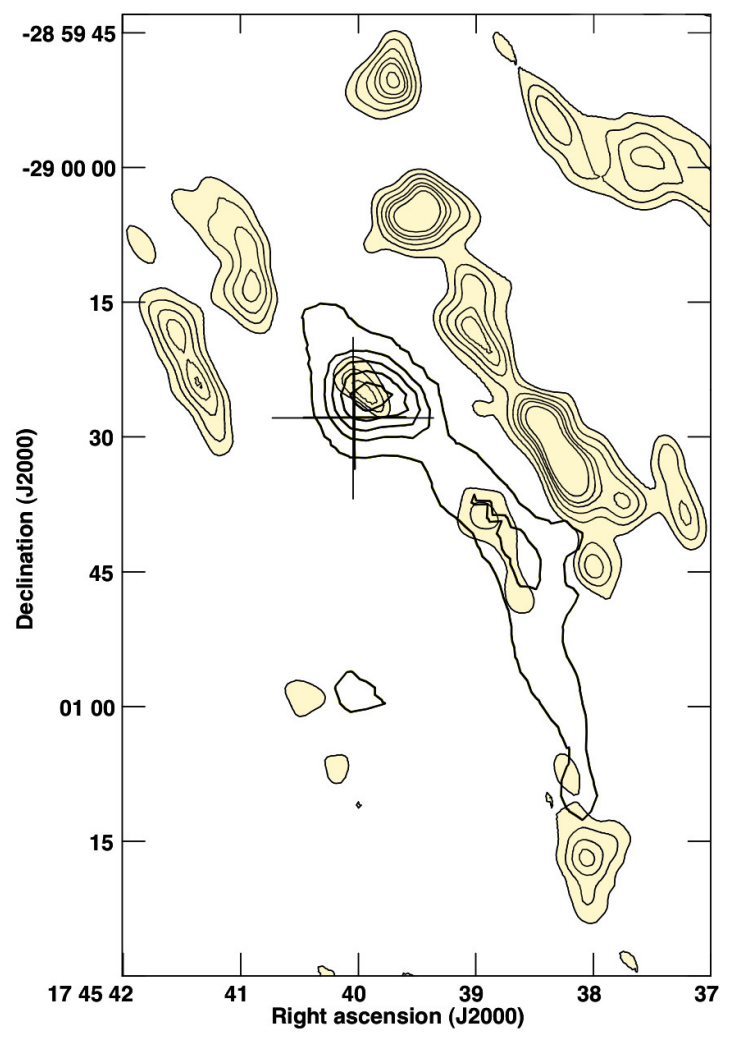

Fig. 5. Contour plot of $\mathrm{CN} J=2-1$ integrated emission between 15 and $45 \mathrm{~km} \mathrm{~s}^{-1}$ (shadowed areas; Martín et al. 2012), with overlay of $\mathrm{OH}$ absorption at $50 \mathrm{~km} \mathrm{~s}^{-1}$ at $1667 \mathrm{MHz}$. Three $\mathrm{CN} J=2-1$ blobs fall inside, or partly inside, of the $\mathrm{OH}$-streamer, and one is immediately $\mathrm{S}$ of the tail. The lowest contour level of $\mathrm{CN} J=2-1$ emission is $\sim 3 \sigma$, and $\sim 4 \sigma$ of OH. Sgr A* is marked with a plus sign. The $\mathrm{CN}$ emission and the head and mid parts of the $\mathrm{OH}$-streamer are seemingly parallel to the Galactic plane.

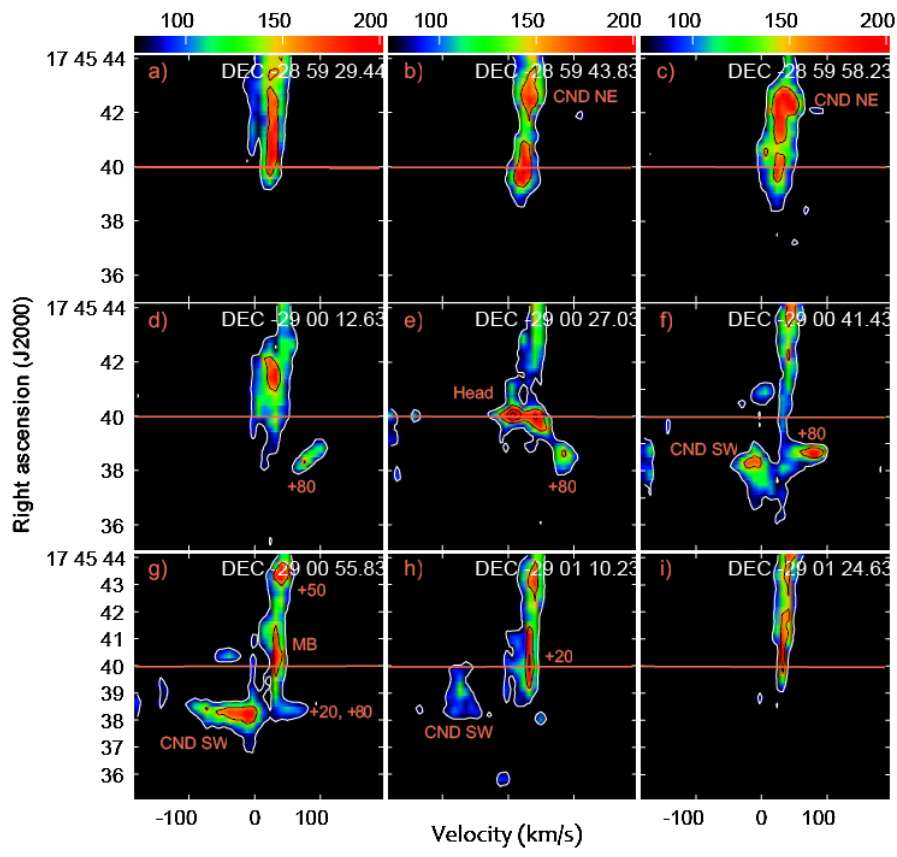

Fig. 6. Position-velocity diagrams (RA, Vel) of $\mathrm{OH}$ absorption at $1667 \mathrm{MHz}$ of an $\sim 2^{\prime} \times 2^{\prime}$ region around $\mathrm{Sgr} \mathrm{A}^{*}$. The lowest contour level is $75 \mathrm{mJy} /$ beam $(\sim 3 \sigma)$ and the contour spacing is also $\sim 3 \sigma$. The horizontal line indicates the right ascension of $\operatorname{Sgr~A}^{*}$, and panel e) is drawn at the declination of Sgr A*. ("MB" stands for molecular belt.) 


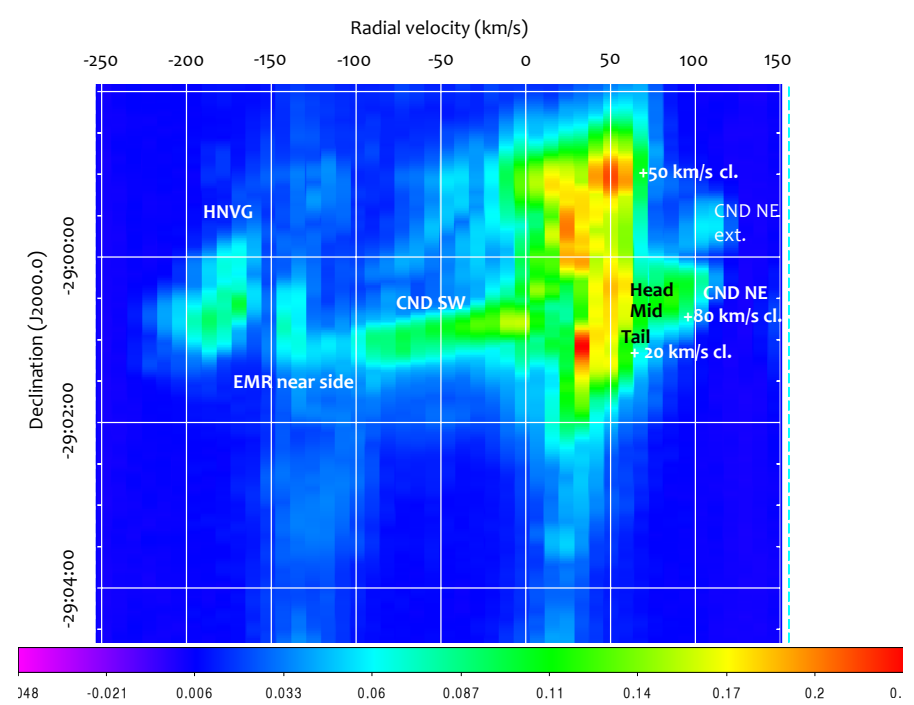

Fig. 7. Position-velocity diagram (Dec, Vel) of $\mathrm{OH}$ absorption at $1667 \mathrm{MHz}$. This is a visualisation through the entire data cube as seen from the declination-velocity side. The $1665 \mathrm{MHz}$ data overlap at velocities higher than about $160 \mathrm{~km} \mathrm{~s}^{-1}$ (see Fig. 3 in Sandqvist 1973). The wedge scale indicates the $\mathrm{OH}$ absorption in Jy/beam. ("HNVG" stands for high negative-velocity gas.)

this interaction is located on the southeastern side of the $\mathrm{OH}-$ streamer tail.

iii) Position angle-velocity diagram: Fig. 8 is a modified diagram by Martín et al. (2012), their Fig. 9, which shows measured component velocities in $\mathrm{CN} J=2-1$ emission as a function of position angle (PA) measured from the position of $\mathrm{Sgr} \mathrm{A}^{*}$. In this figure we have suppressed all points observed by Martin et al. (2012), except for those belonging to their rotating partial ring models for the CND, and superimposed our own calculated velocities and PAs for the OH-streamer and the $+80 \mathrm{~km} \mathrm{~s}^{-1}$ cloud at Positions 1-16 (Fig. A.1 and Table A.1).

The OH-streamer shows a nearly flat velocity structure of about $50 \mathrm{~km} \mathrm{~s}^{-1}$ in the head between PAs from about $260^{\circ}$ to $310^{\circ}$ in Fig. 8. In the higher resolution BnA observations in Fig. 3, we find that a winglike structure of the head occurs at $50 \mathrm{~km} \mathrm{~s}^{-1}$ and persists to $-3 \mathrm{~km} \mathrm{~s}^{-1}$, while displaying an anti-clockwise rotation (the arrows in Fig. 3), and may be a signature of rotation of the $\mathrm{OH}$ gas in the head about $\mathrm{Sgr} \mathrm{A}^{*}$ or infalling of gas towards $\mathrm{Sgr} \mathrm{A}^{*}$. The $\mathrm{OH}$ absorption also declines while the velocity drops from $50 \mathrm{~km} \mathrm{~s}^{-1}$ to $-20 \mathrm{~km} \mathrm{~s}^{-1}$. In Fig. 8, the velocity is seen to increase about $10 \mathrm{~km} \mathrm{~s}^{-1}$ between PAs of about $230^{\circ}$ and $260^{\circ}$ corresponding to the mid part of the $\mathrm{OH}$-streamer, which is also the overlap region of the mid part and the $+80 \mathrm{~km} \mathrm{~s}^{-1}$ cloud. The velocity of the two outermost points in the tail decreases towards the values in the $+20 \mathrm{~km} \mathrm{~s}^{-1}$ cloud; this region is indicated with an ellipse in Fig. 8.

The $+80 \mathrm{~km} \mathrm{~s}^{-1}$ cloud is sampled well at PAs between $250^{\circ}$ and $315^{\circ}$ in Fig. 8, a similar interval as the "70 $\mathrm{km} \mathrm{s}^{-1}$ sources" observed in CN emission (Martín et al. 2012), and it appears that those sources are located in the $+80 \mathrm{~km} \mathrm{~s}^{-1}$ cloud. It is clear from this figure that the dominant part of the $+80 \mathrm{~km} \mathrm{~s}^{-1}$ cloud is not part of the western edge of the CND. However, its northernmost part might be connecting to the CND rotating ring model 1 in the sector of PAs about $320^{\circ}$ to $330^{\circ}$.

The $+20 \mathrm{~km} \mathrm{~s}^{-1}$ cloud shows a flat velocity signature from PAs of $180^{\circ}$ to $280^{\circ}$, after which the velocity appears to approach that of the CND rotating ring model 3, near a PA of $310^{\circ}$.

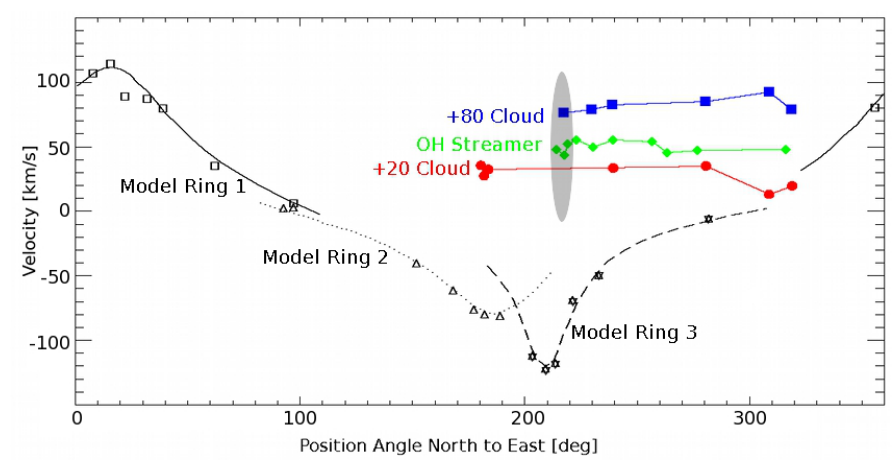

Fig. 8. Nominal velocities as a function of position angle $\mathrm{E}$ of $\mathrm{N}$, as measured from the position of $\mathrm{Sgr} \mathrm{A}^{*}$, of the OH-streamer (green filled diamonds), the $+80 \mathrm{~km} \mathrm{~s}^{-1}$ cloud (blue filled squares), and the $+20 \mathrm{~km} \mathrm{~s}^{-1}$ cloud (red filled circles) overlaid on a diagram by Martín et al. (2012), which shows the location of selected objects observed in $\mathrm{CN}$ emission. The ellipse indicates the region where interaction between the +20 , $+80 \mathrm{~km} \mathrm{~s}^{-1}$ clouds and the $\mathrm{OH}$-streamer is suggested. The overlaid sinusoidal-like lines represent rotating ring models fitted to the core velocities in the southwest lobe (dashed line), southern extension (dotted line), and northeast lobe of the CND (solid line).

\section{Concatenated $\mathrm{OH}$ data cubes}

Spectral line maps of $\mathrm{OH}$ absorption at $1667 \mathrm{MHz}$ with an angular resolution of $7^{\prime \prime} \times 5^{\prime \prime}$ are provided in Appendix B at velocities between -196 and $111 \mathrm{~km} \mathrm{~s}^{-1}$. The corresponding $-T_{\mathrm{L}} / T_{\mathrm{C}}$ $\mathrm{OH}$ maps at $1667 \mathrm{MHz}$ are presented in Appendix C. As a representative of the $1665 \mathrm{MHz}$ OH maps, we have chosen a few maps at velocities between 15 and $59 \mathrm{~km} \mathrm{~s}^{-1}$ which are presented in Appendices D and E. This limited velocity range covers some of the more pertinent components in this paper.

In additon to the objects discussed in this paper, many other known objects, such as the foreground spiral arm features at velocities of about $-50,-30$, and $0 \mathrm{~km} \mathrm{~s}^{-1}$, the high negative velocity gas, and the wide velocity feature (Karlsson et al. 2013), can be seen in those maps.

Although much additional information can be gleaned from the maps, we wish here to restrict ourselves to an update concerning the compact $\mathrm{H}$ II regions (A-D; Ekers et al. 1983) on the eastern edge of the $+50 \mathrm{~km} \mathrm{~s}^{-1}$ cloud. In Karlsson et al. (2013), we reported observations of $\mathrm{OH}$ absorption against the compact $\mathrm{H}$ II region D. A further analysis of the data now reveals that $\mathrm{OH}$ absorption is also seen against the three other regions, A, B, and $\mathrm{C}$, in the velocity ranges of 24 to $76 \mathrm{~km} \mathrm{~s}^{-1}$ and 32 to $76 \mathrm{~km} \mathrm{~s}^{-1}$, for the 1667 and $1665 \mathrm{MHz}$ lines, respectively (Figs. C.1, C.2, D.1, and E.1).

The four complete data cubes of the $\mathrm{OH}$ absorption and $-T_{\mathrm{L}} / T_{\mathrm{C}}$ at 1665 and $1667 \mathrm{MHz}$ are available in electronic form at the $\mathrm{CDS}^{2}$.

\section{Discussion}

The OH-streamer is clearly observed between velocities of -29 and $67 \mathrm{~km} \mathrm{~s}^{-1}$. At velocities between 0 and $10 \mathrm{~km} \mathrm{~s}^{-1}$ confusion with foreground sources may occur. The $\mathrm{OH}$-streamer is not observed in the $-T_{\mathrm{L}} / T_{\mathrm{C}}$ maps at velocities less than $41 \mathrm{~km} \mathrm{~s}^{-1}$. Clipping of the different random noise levels in the channel and continuum maps and absorption in foreground objects may explain the absence of this feature at those velocities in the $-T_{\mathrm{L}} / T_{\mathrm{C}}$ maps.

2 At http://vizier.u-strasbg.fr/viz-bin/VizieR 
Species like $\mathrm{CN}$ and $\mathrm{OH}$ tend to be abundant in photo dissociation regions, suggesting that they mark the regions where UV radiation from the central star cluster interacts with the surrounding neutral gas. The $\mathrm{OH}$ column densities divided by excitation temperatures $\left(N(\mathrm{OH}) / T_{\text {ex }}\right)$ for the different parts are given in Table A.1. It is likely that $T_{\text {ex }}$ increases with decreasing distance from Sgr $\mathrm{A}^{*}$, which would imply increasing $\mathrm{OH}$ column densities towards the head.

In the region of the head, UV-radiation pressure from the central star cluster may be a factor that could lead to outflow of $\mathrm{OH}$ gas from the head. However, Murray et al. (2011) find that outflows from clusters in a Milky way-like galaxy (their Fig. 4), would lead to outflow velocites of several hundreds of $\mathrm{km} \mathrm{s}^{-1}$, at distances from the centre relevant for the $\mathrm{OH}$-streamer head $(0.3 \mathrm{pc})$, where we observe velocities of about $50 \mathrm{~km} \mathrm{~s}^{-1}$. At distances less than about $0.1 \mathrm{pc}$, the radiation pressure may, however, be significant in supporting self-gravitational disks that are supposed to fuel the SMBH at the centre (Thompson 2009). We therefore argue that radiation pressure is not significant for the kinematics of the head.

The influence radius of the SMBH and the surrounding star cluster can be estimated by calculating the total gravitational specific force in its surrounding few parsecs. Following Sanders (1998) and assuming a core radius of $0.085 \mathrm{pc}$ and the mass of the $\mathrm{SMBH}$ to be $4.5 \times 10^{6} M_{\odot}$, it becomes clear that the force from the SMBH and the surrounding star cluster is significant at distances less than about $0.25 \mathrm{pc}\left(\sim 6^{\prime \prime}\right)$, i.e. in the region of the $\mathrm{OH}$-streamer head. It decreases by more than one order of magnitude at distances greater than about $0.7 \mathrm{pc}$ where the mid and tail parts of the $\mathrm{OH}$-streamer reside. Thus, the gravitational pull of the SMBH and the surrounding star cluster is strong enough to affect the kinematics of the head, but not the mid or tail.

In a recent study, Yusef-Zadeh et al. (2013) identified blobs of weak radio emission at $8.4 \mathrm{GHz}$ located on a line through the position of $\mathrm{SgrA}^{*}$ at a PA of $\sim 30^{\circ} \mathrm{E}$ of N. Three of the blobs, b), c), and d), appear to be accommodated inside of the $\mathrm{OH}$-streamer between the head and mid maxima. Furthermore, $\mathrm{CN} J=2-1$ emission is observed at the position of blob d) (RA, Dec $)=17^{\mathrm{h}} 45^{\mathrm{m}} 38^{\mathrm{s}} .8,-29^{\circ} 00^{\prime} 40^{\prime \prime}$. We note that this position also coincides with the region of intersection between the eastern part of the $+80 \mathrm{~km} \mathrm{~s}^{-1}$ cloud and the mid part of the $\mathrm{OH}$-streamer, i.e. where a steep intensity gradient is seen in Fig. 4.

The $+80 \mathrm{~km} \mathrm{~s}^{-1}$ cloud interacts with the $\mathrm{OH}$-streamer and the CND, while the cloud is not a part of the CND itself. The southern part of the cloud interacts with the OH-streamer mid and tail parts, as seen both in the map- and positionvelocity planes. Interaction between the $\mathrm{OH}$-streamer and the $+80 \mathrm{~km} \mathrm{~s}^{-1}$ cloud in this region may also be indicated by the striking increase in the value of $\Delta V_{\mathrm{FWHM}}$ of line profile components here. Although shock-excited 1720-MHz OH maser sources were not found in 1985 in this region of interaction (Karlsson et al. 2003), it may well be worthwhile repeating such a search at a later epoch. The velocity in the northern part of the $+80 \mathrm{~km} \mathrm{~s}^{-1}$ cloud seems to be generally increasing to the north and to adapt to the velocity of the CND NE lobe as observed in $\mathrm{CN}$ emission. This part of the cloud may, however, be a detached clump of the $+80 \mathrm{~km} \mathrm{~s}^{-1}$ cloud (Figs. B.1 and C.1).

Interaction between the $+20 \mathrm{~km} \mathrm{~s}^{-1}$ cloud and the CND SW lobe (via the SS) is consistent with the observation of Coil \& Ho (1999) that the northern tip of the $+20 \mathrm{~km} \mathrm{~s}^{-1}$ cloud/(SS) interacts with (feeds) the CND SW lobe. Additionally, Karlsson et al. (2013), observing this region in the $\mathrm{C}^{18} \mathrm{O} J=2-1$ emission line, found that the northern part of the $+20 \mathrm{~km} \mathrm{~s}^{-1}$ cloud/SS has an extension which bends abruptly from the northwest to the northeast, pointing in towards the $\mathrm{OH}$-streamer tail and CND SW lobe (see their Fig. 11). The presence of shock activity in this common region of the $\mathrm{OH}$-streamer, the +20 and $+80 \mathrm{~km} \mathrm{~s}^{-1}$ clouds, and the CND SW is furthermore supported by recent Odin observations of an unusually wide $\mathrm{H}_{2}^{18} \mathrm{O}$ positive-velocity absorption line. The abundance ratio of $\mathrm{H}_{2} \mathrm{O}$ with respect to $\mathrm{H}_{2}$ was found to be approximately $1.4 \times 10^{-6}$, a high value indicative of the presence of strong shocks desorbing water from dust grains in this region (Karlsson et al. 2013, and Appendix F). In Fig. 8, we have tentatively indicated the region of interaction (in the map plane) between the +20 and $+80 \mathrm{~km} \mathrm{~s}^{-1}$ clouds and the $\mathrm{OH}$-streamer.

A new result in our data is that of a possible link between the near side of the EMR and the CND SW lobe (Figs. 7 and A.3). Is the EMR a link in the process of transporting material from the $100 \mathrm{pc}$ scale to the $10 \mathrm{pc}$ scale of the CND via molecular clouds (Fathi et al. 2006)? Incidentally, this region also coincides with the rotating ring model of the CND where the velocity is about $-115 \mathrm{~km} \mathrm{~s}^{-1}$ with a velocity width of $40 \mathrm{~km} \mathrm{~s}^{-1}$ (Martín et al. 2012). Furthermore, Emsellem et al. (2014) have studied the interplay between a galactic bar and an SMBH by simulating a Milky Way-like galaxy. They found that gas is focused on (Lindblad) resonances into elongated ring-like structures and subsequently connects to the SMBH via mini-spirals inside of a few tens of pc. Ultimately, the gas accreted in the vicinity of the SMBH creates a series of winding tails of gas, supporting our results in this work. In particular, the $\mathrm{OH}$-streamer resembles the features presented in Emsellem et al. (2014), which suggest that it could have been triggered by tidal-like forces owing to the interplay between the gravitational potential of the Galactic bar and that of the SMBH at the centre of the Galaxy.

\section{Conclusions}

We have presented an analysis of certain features in our concatenated data base of VLA BnA and DnC observations of 1665 and $1667 \mathrm{MHz} \mathrm{OH}$ absorption towards the Sgr A Complex at the GC. Our data have an angular resolution of about $6^{\prime \prime}$ and a velocity resolution of about $9 \mathrm{~km} \mathrm{~s}^{-1}$, and the full data set has been made available to the scientific community using the $\mathrm{CDS}^{3}$.

Our investigation of the properties and kinematics of the $\mathrm{OH}$-streamer and the $+80 \mathrm{~km} \mathrm{~s}^{-1}$ cloud in $\mathrm{OH}$ absorption indicated that the OH-streamer is an object inside of the CND and interacting with the $+80 \mathrm{~km} \mathrm{~s}^{-1}$ cloud, the CND, and possibly with the strong gravitational field from the SMBH and the surrounding star cluster. We also found indications of interaction between the $+80 \mathrm{~km} \mathrm{~s}^{-1}$ cloud, or a detached clump of it, and the northeastern lobe of the CND via the CND NE extension.

We interpreted those kinematical and morphological links as indications that gas clumps were disrupted from the SW lobe of the CND and may have produced the $\mathrm{OH}$-streamer and the $+80 \mathrm{~km} \mathrm{~s}^{-1}$ cloud. At least the $\mathrm{OH}$-streamer seems to be feeding material radially inwards, inside of the CND. For the $+80 \mathrm{~km} \mathrm{~s}^{-1}$ cloud the kinematics have still not been resolved. Although the projected image of this cloud may suggest that it is a part of the $\mathrm{CND}$, the position-velocity diagrams clearly dispute this.

Our conclusions are that the $\mathrm{OH}$-streamer head is located in front of $\mathrm{Sgr} \mathrm{A}^{*}$ at velocities between about 15 and $50 \mathrm{~km} \mathrm{~s}^{-1}$ and partly behind at velocities between 59 and $67 \mathrm{~km} \mathrm{~s}^{-1}$ and that the $\mathrm{OH}$-streamer head represents a part of an inflow of gas from the CND region. In the mid and tail parts, as well as in the head, we note a negative velocity gradient from the $+80 \mathrm{~km} \mathrm{~s}^{-1}$

http://vizier.u-strasbg.fr/viz-bin/VizieR 
cloud and towards the GC, where one would expect increasing velocity inwards from the CND. If the $\mathrm{OH}$-streamer and the $+80 \mathrm{~km} \mathrm{~s}^{-1}$ cloud are located on the "far" side of the CND and behind Sgr A West, a flow from "behind" towards the centre would display such a negative velocity gradient.

Moreover, a detailed analysis of the position-velocity diagrams revealed a possible link between the near side of the EMR and the CND SW lobe. In this analysis we also found $\mathrm{OH}$ absorption against all four of the compact $\mathrm{H}$ II regions $\mathrm{A}-\mathrm{D}$, east of Sgr A East, both in the 1665 and $1667 \mathrm{MHz}$ transitions.

Further progress on the matter of relative locations and relations between the $\mathrm{OH}$-streamer, the $+80 \mathrm{~km} \mathrm{~s}^{-1}$ cloud, and the surrounding molecular clouds and Sgr $\mathrm{A}^{*}$ would be gained from observations of $\mathrm{OH}$ with still higher angular and velocity resolutions and from searches for those objects in other species. Furthermore, a state-of-the-art 3D modelling would be highly beneficial to reveal the relative locations of molecular clouds in the $10 \mathrm{pc}$ region surrounding the GC.

Acknowledgements. The authors acknowledge the open policy for the use of NRAOs VLA. The National Radio Astronomy Observatory (NRAO) is operated by Associated Universities Inc., under cooperative agreement with the National Science Foundation. Kambiz Fathi acknowledges support from the Swedish Royal Academy of Sciences' Crafoord Foundation. Furthermore, the extensive support from the Swedish National Space Board (Rymdstyrelsen) is gratefully acknowledged by Aage Sandqvist. We also acknowledge with gratitude the support from Miller Goss with concatenation of the observations with the VLA in its BnA and DnC configurations.

\section{References}

Bieging, J. H. 1976, A\&A, 51, 289

Coil, A. L., \& Ho, P. T. P. 1999, ApJ, 513, 752

Ekers, R. D., van Gorkom, J. H., Schwarz, U. J., \& Goss, W. M. 1983, A\&A, 122,143

Emsellem, E., Renaud, F., Frédéric, B., et al. 2015, MNRAS, 446, 2468

Fathi, K., Storcgi-Bergmann, T., Riffel, R. A., et al. 2006, ApJ, 641, L25

Ferrière, K. 2012, A\&A, 540, A50

Goldsmith, P. F. 1999, Millimeter-Wave Astronomy: Molecular Chemistry \& Physics in Space, eds. W. F. Wall, et al., ASSL, 241, 57

Karlsson, R., Sjouwerman, L. O., Sandqvist, A., \& Whiteoak, J. B. 2003, A\&A, 403, 1011

Karlsson, R., Sandqvist, A., Hjalmarson, Å, et al. 2013, A\&A, 554, A141

Kerr, F. J., \& Sandqvist, A. 1968, Astrophys. Lett., 2, 195

Killeen, N. E. B., Lo, K. Y., \& Crutcher, R. 1992, ApJ, 385, 585

Liu, H. B., Hsieh, P.-Y., Ho, P. T. P., et al. 2012, ApJ, 756, 195

Martín, S., Martín-Pintado, J., Montero-Castaño, M., et al. 2012, A\&A, 539, A29

Mezger, P. G., Duschl, W. J., \& Zylka, R. 1996, A\&AR, 7, 289

Morris, M., \& Serabyn, E. 1996, ARA\&A, 34, 645

Murray, N., Ménard, B., \& Thompson, T. A. 2011, ApJ, 735, 66

Persson, C. M., Olofsson, A. O. H., Koning, N., et al. 2007, A\&A, 476, 807

Sanders, R. H. 1998, MNRAS, 294, 35

Sandqvist, A. 1973, A\&AS, 9, 391

Sandqvist, A. 1974, A\&A, 33, 413

Sandqvist, A., Karlsson, R., Whiteoak, J. B., \& Gardner, F. F. 1987, in The Galactic Center, ed. D. C. Backer, AIP Conf. Proc., 155, 95

Sandqvist, A., Karlsson, R., \& Whiteoak, J. B. 1989, in The Center of the Galaxy, ed. M. Morris (Kluwer), IAU Symp., 136, 421

Thompson, T. A. 2009, ASP Conf. Ser., 408, 128

Wilson, T. L., \& Rood, R. 1994, ARA\&A, 32, 191

Yusef-Zadeh, F., Royster, M., Wardle, M., et al. 2013, ApJ, 767, L32 


\section{Appendix A: OH absorption}

Figure A.1 is a map that indicates the positions where the profiles in Fig. A. 2 were produced using the $1667-\mathrm{MHz} \mathrm{OH}$ absorption data cube. Positions 1-4 are located in the $\mathrm{OH}$-streamer head, $5-7$ in the mid, and $8-10$ in the tail part. Positions $11-13$ are in the $+80 \mathrm{~km} \mathrm{~s}^{-1}$ cloud, and positions 14-16 are in the northern part of the $+20 \mathrm{~km} \mathrm{~s}^{-1}$ cloud. The $\mathrm{OH}$ parameters, obtained from a Gaussian analysis of these profiles, are presented in Table A.1.

Figure A.3 is a position-velocity diagram of the $\mathrm{OH}$ absorption at $1667 \mathrm{MHz}$ of the inner $7^{\prime} \times 7^{\prime}$ region of the GC. It is a visualisation through the entire data cube seen face-on from the right ascension-velocity side. The angular resolution is $7^{\prime \prime}$, and the velocity resolution is $8.8 \mathrm{~km} \mathrm{~s}^{-1}$. The prominent features are labelled in the figure. We note the bridge between the EMR near side and the CND SW lobe.

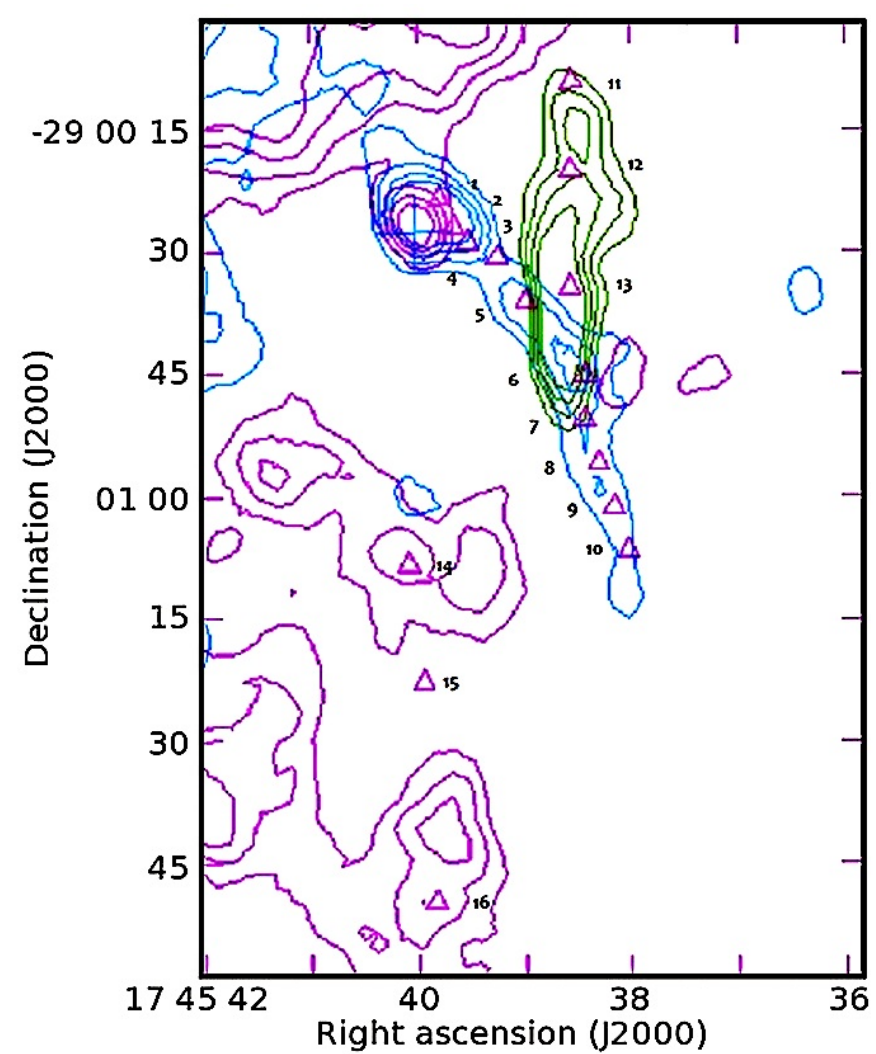

Fig. A.1. Locations of the $\mathrm{OH}$ absorption profiles, labelled by triangles. The magenta contours indicate the $\mathrm{OH}$-streamer, the olive-coloured contours depict the +80 cloud, and the purple contours delineate parts of the $+20 \mathrm{~km} \mathrm{~s}^{-1}$ cloud, at 50,85 , and $32 \mathrm{~km} \mathrm{~s}^{-1}$, respectively. The lowest contour level is at $3.5 \sigma(90 \mathrm{mJy} / \mathrm{beam})$, and the contour interval is $1 \sigma$ spacing. Position numbers $1-10$ belong to the $\mathrm{OH}$-streamer, 11-13 are in the $+80 \mathrm{~km} \mathrm{~s}^{-1}$ cloud, and 14-16 are inside the $+20 \mathrm{~km} \mathrm{~s}^{-1}$ cloud.

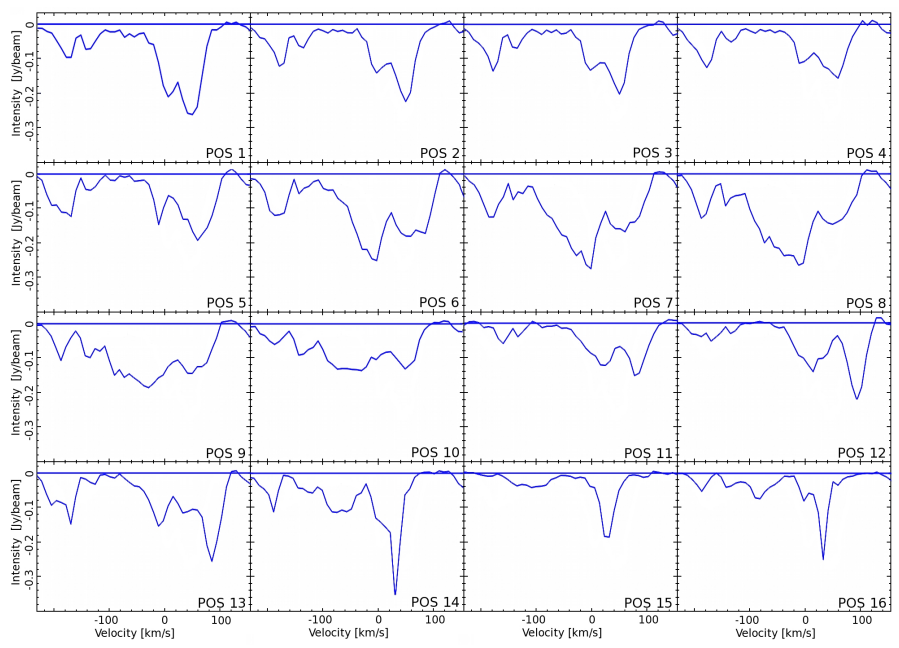

Fig. A.2. 1667-MHz OH absorption line profiles at the positions shown in Fig. A.1. The angular resolution is $7^{\prime \prime} \times 7^{\prime \prime}$, and the velocity resolution is $8.8 \mathrm{~km} \mathrm{~s}^{-1}$.

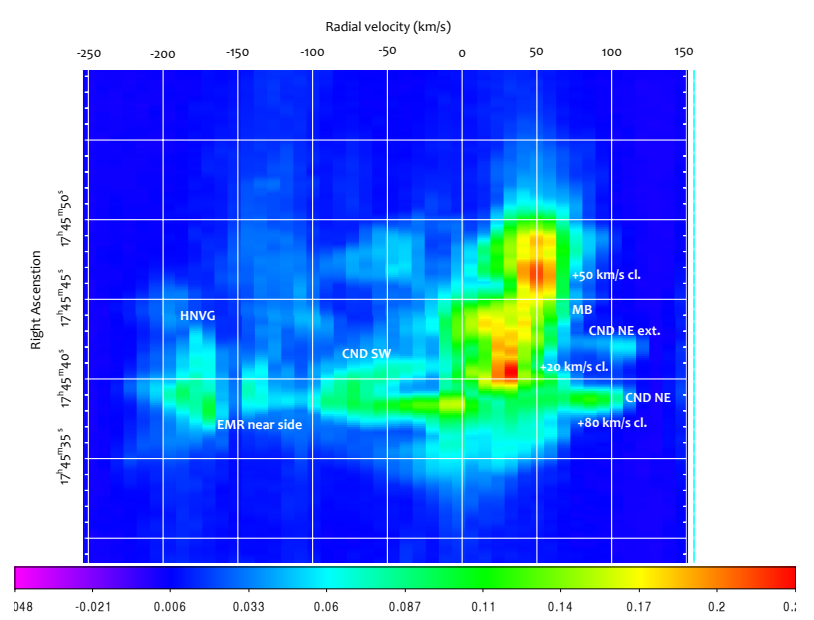

Fig. A.3. Position-velocity diagram (RA, Vel) of $\mathrm{OH}$ absorption at $1667 \mathrm{MHz}$. This is a visualisation through the entire data cube as seen from the right ascension-velocity side. The $1665 \mathrm{MHz}$ data overlap at velocities higher than about $160 \mathrm{~km} \mathrm{~s}^{-1}$, see Fig. 3 in Sandqvist (1973). The wedge scale indicates the $\mathrm{OH}$ absorption in Jy/beam. ("HNVG" stands for high negative velocity gas, "MB" the molecular belt.) 
R. Karlsson et al.: OH in GC molecular clouds

Table A.1. Parameters obtained from the Gaussian decomposition of the profiles at Positions 1-16 in Fig. A.1.

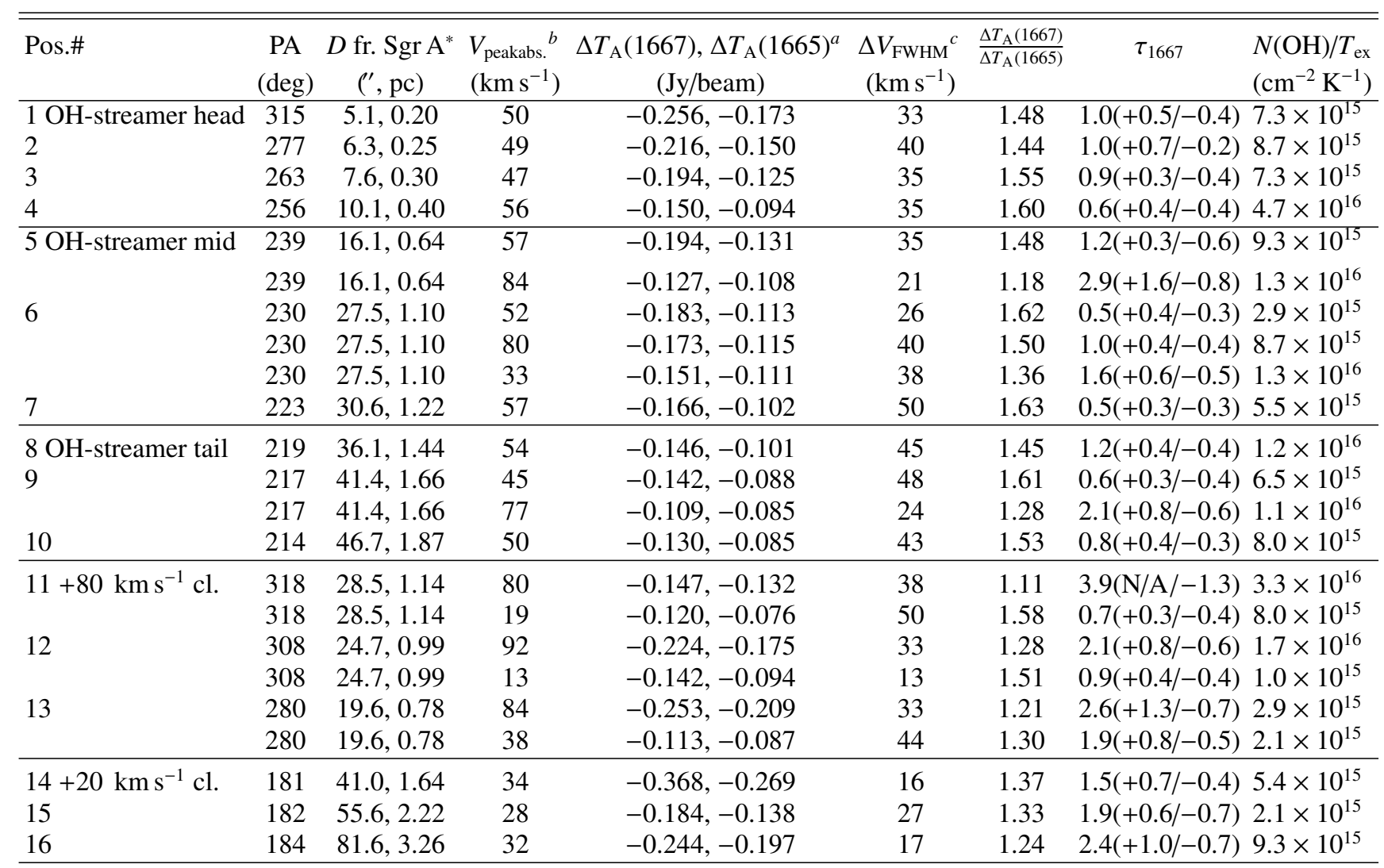

Notes. ${ }^{(a)}$ Intensities $>3 \sigma,{ }^{(b)}\left(V_{\text {peak abs. }}\right)=$ velocity at maximum absorption, ${ }^{(c)}\left(\Delta V_{\text {FWHM }}\right)=$ linewidth. 
Appendix B: $1667 \mathrm{MHz}$ OH-absorption

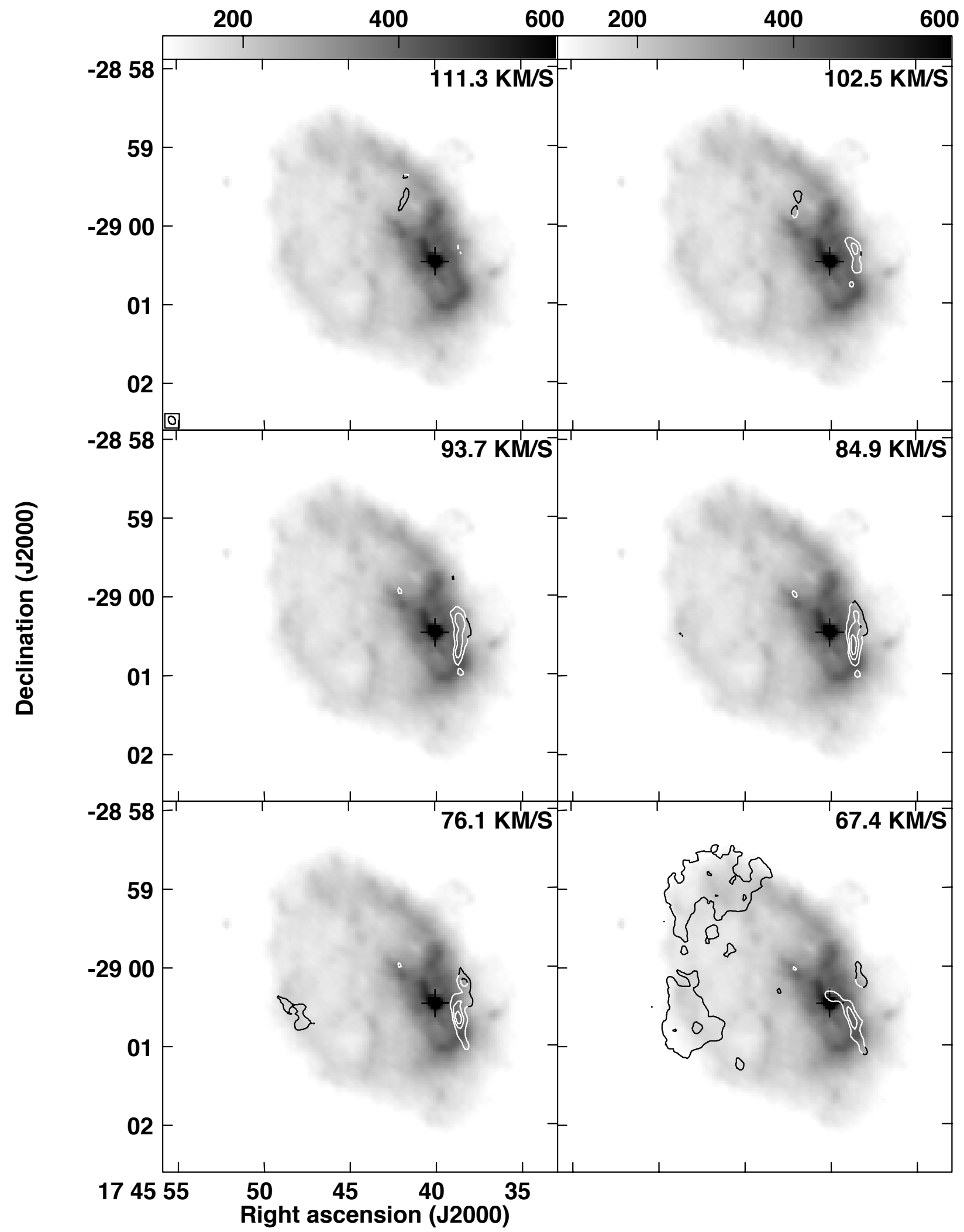

Fig. B.1. 1667-MHz OH-absorption at $111>V_{\mathrm{LSR}}>67 \mathrm{~km} \mathrm{~s}^{-1}$. The lowest contour level is $50 \mathrm{mJy} /$ beam $(\sim 2 \sigma)$ and the contour spacing is also $\sim 2 \sigma$. The wedge scale is in $\mathrm{mJy} / \mathrm{beam}$. The position of Sgr $\mathrm{A}^{*}$ is marked with a plus sign. 
R. Karlsson et al.: $\mathrm{OH}$ in GC molecular clouds

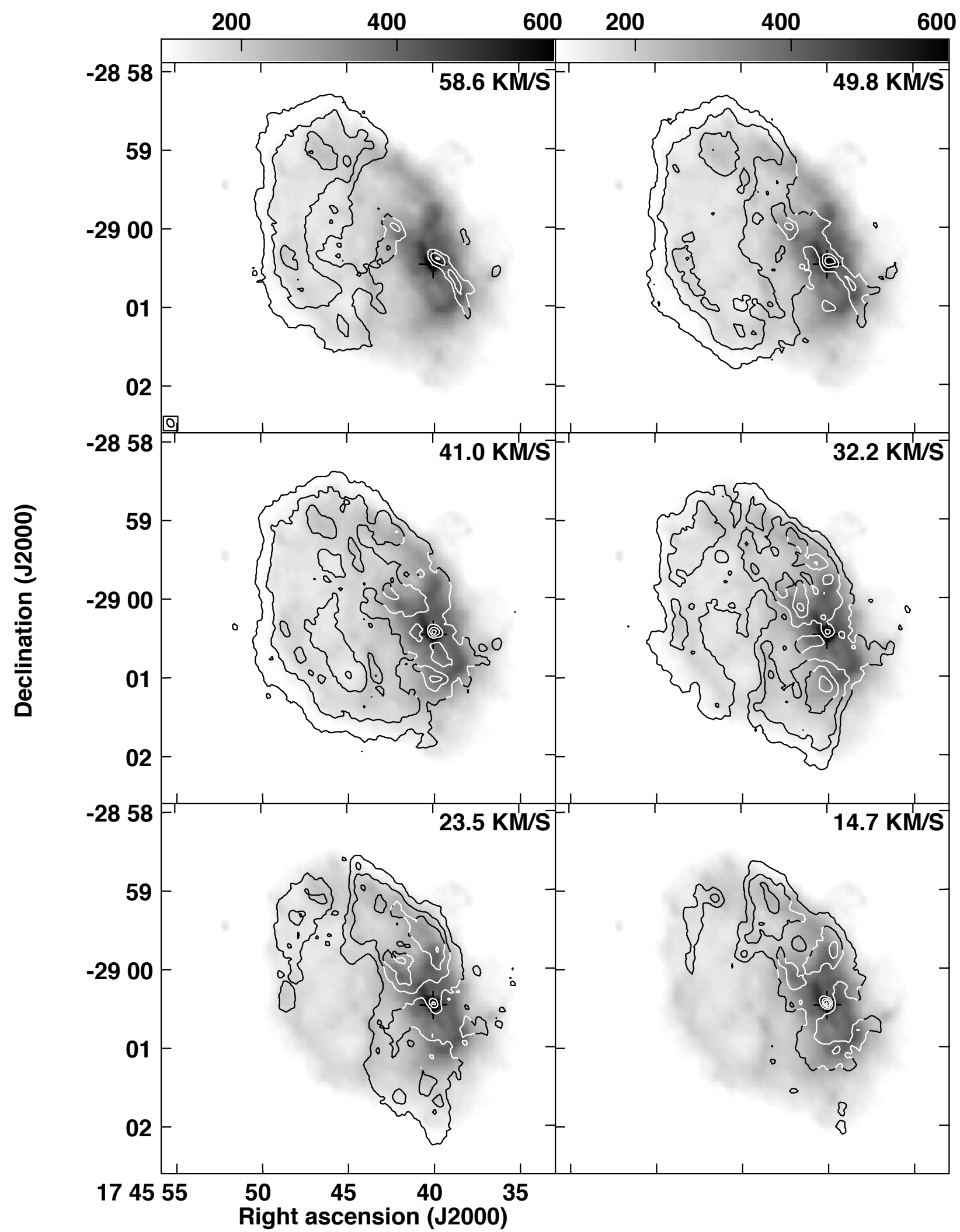

Fig. B.2. 1667-MHz OH-absorption at $59>V_{\mathrm{LSR}}>15 \mathrm{~km} \mathrm{~s}^{-1}$. The lowest contour level is $50 \mathrm{mJy} /$ beam $(\sim 2 \sigma)$, and the contour spacing is also $\sim 2 \sigma$. The wedge scale is in $\mathrm{mJy} / \mathrm{beam}$. The position of $\mathrm{Sgr} \mathrm{A}^{*}$ is labelled with a plus sign. 
A\&A 582, A118 (2015)

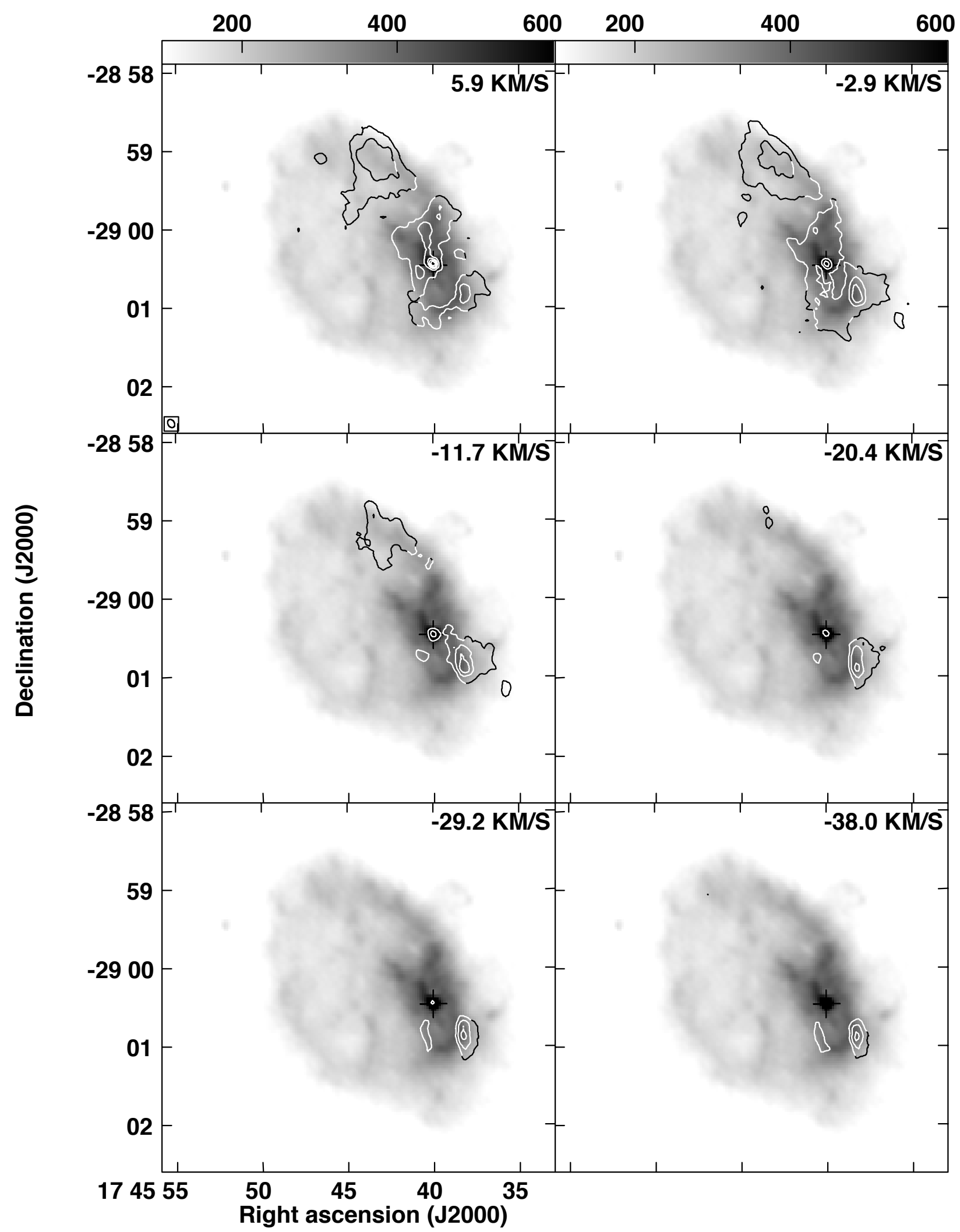

Fig. B.3. 1667-MHz OH-absorption at $6>V_{\mathrm{LSR}}>-38.0 \mathrm{~km} \mathrm{~s}^{-1}$. The lowest contour level is $50 \mathrm{mJy} / \mathrm{beam}(\sim 2 \sigma)$, and the contour spacing is also $\sim 2 \sigma$. The wedge scale is in $\mathrm{mJy} / \mathrm{beam}$. The position of Sgr A* is labelled with a plus sign. 
R. Karlsson et al.: OH in GC molecular clouds

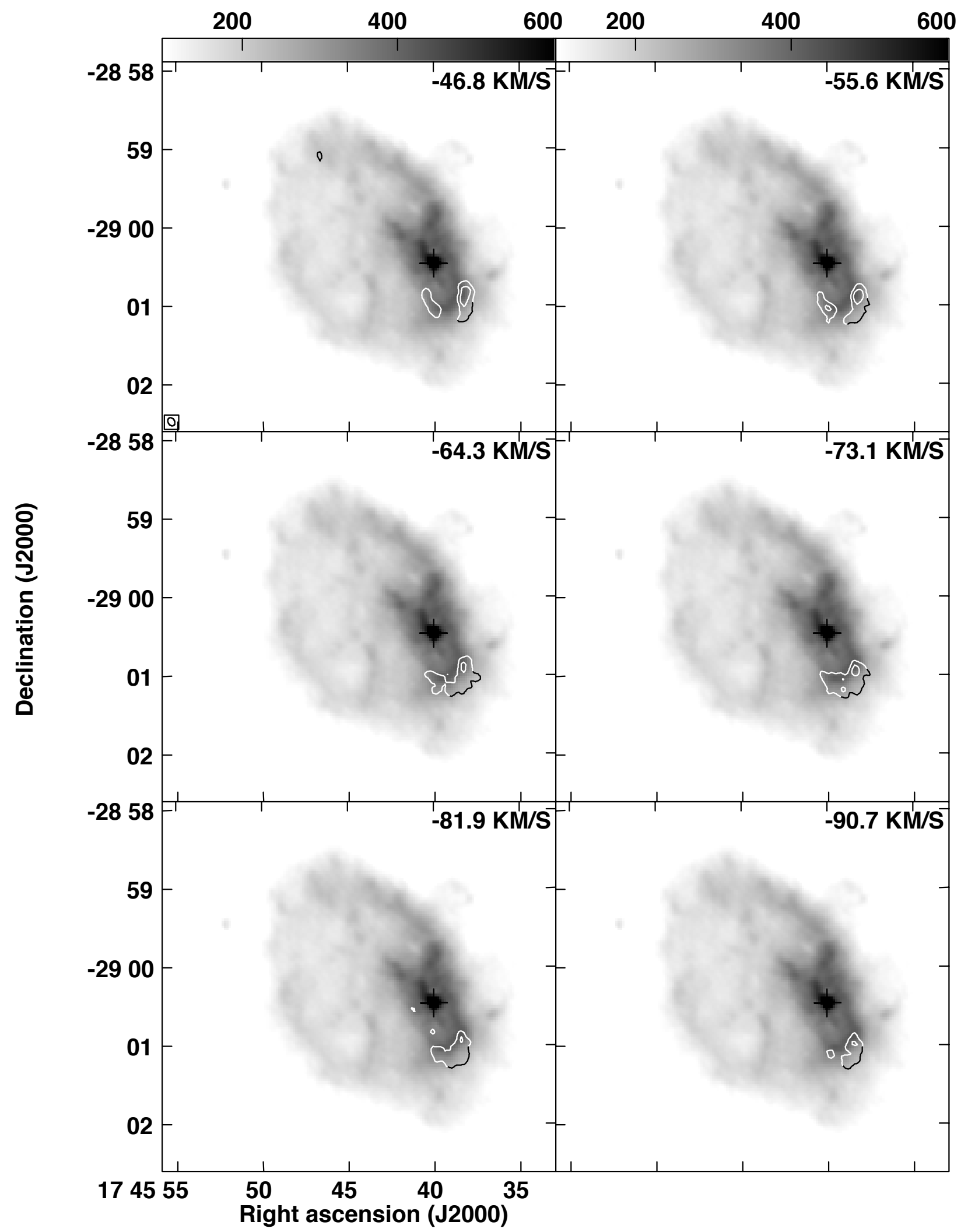

Fig. B.4. 1667-MHz OH-absorption at $-47>V_{\mathrm{LSR}}>-91 \mathrm{~km} \mathrm{~s}^{-1}$. The lowest contour level is $50 \mathrm{mJy} / \mathrm{beam}(\sim 2 \sigma)$, and the contour spacing is also $\sim 2 \sigma$. The wedge scale is in $\mathrm{mJy} / \mathrm{beam}$. The position of $\mathrm{Sgr} \mathrm{A}^{*}$ is labelled with a plus sign. 


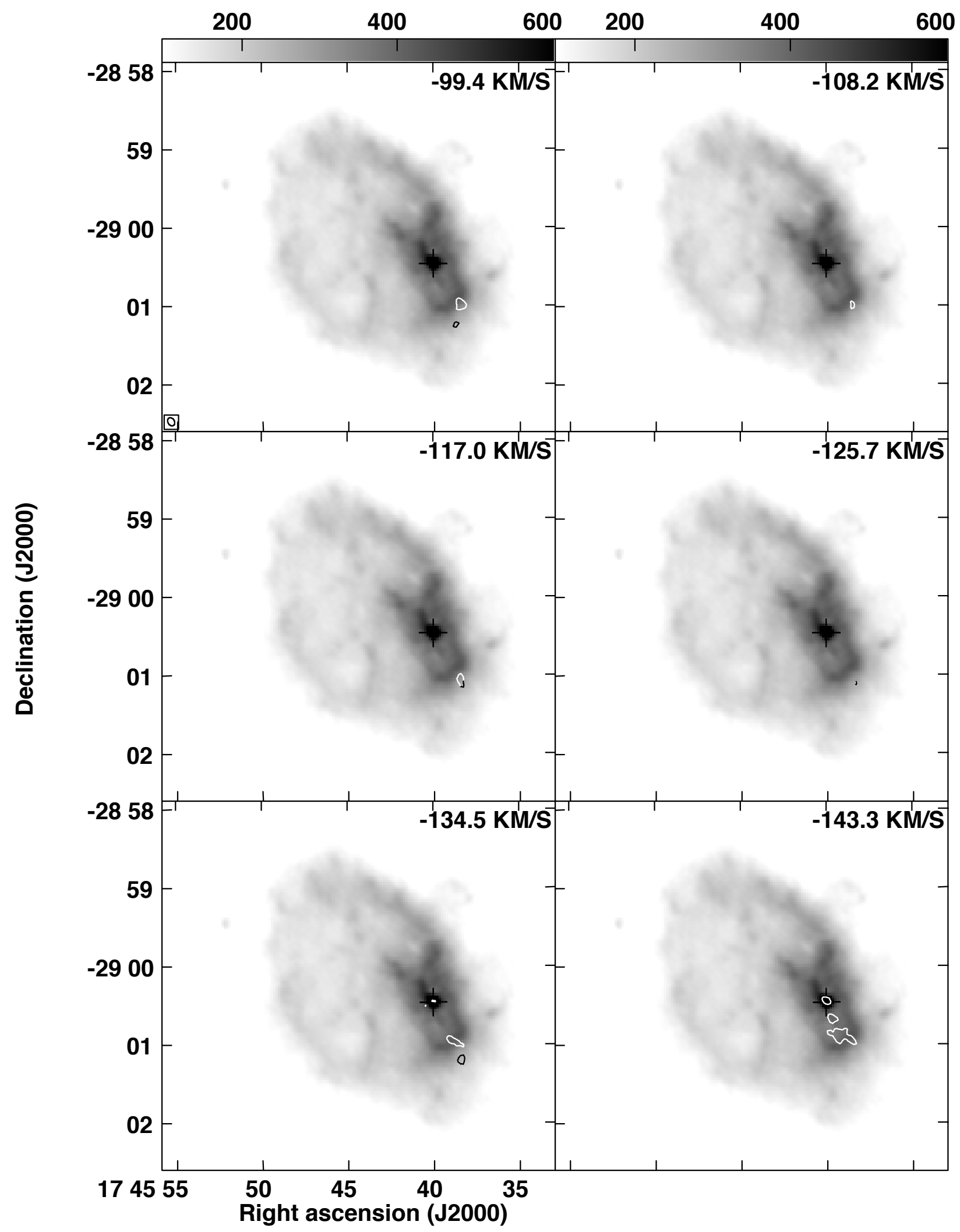

Fig. B.5. 1667-MHz OH-absorption at $-99>V_{\mathrm{LSR}}>-143 \mathrm{~km} \mathrm{~s}^{-1}$. The lowest contour level is $50 \mathrm{mJy} /$ beam $(\sim 2 \sigma)$, and the contour spacing is also $\sim 2 \sigma$. The wedge scale is in $\mathrm{mJy} / \mathrm{beam}$. The position of $\mathrm{Sgr} \mathrm{A}^{*}$ is labelled with a plus sign. 
R. Karlsson et al.: OH in GC molecular clouds

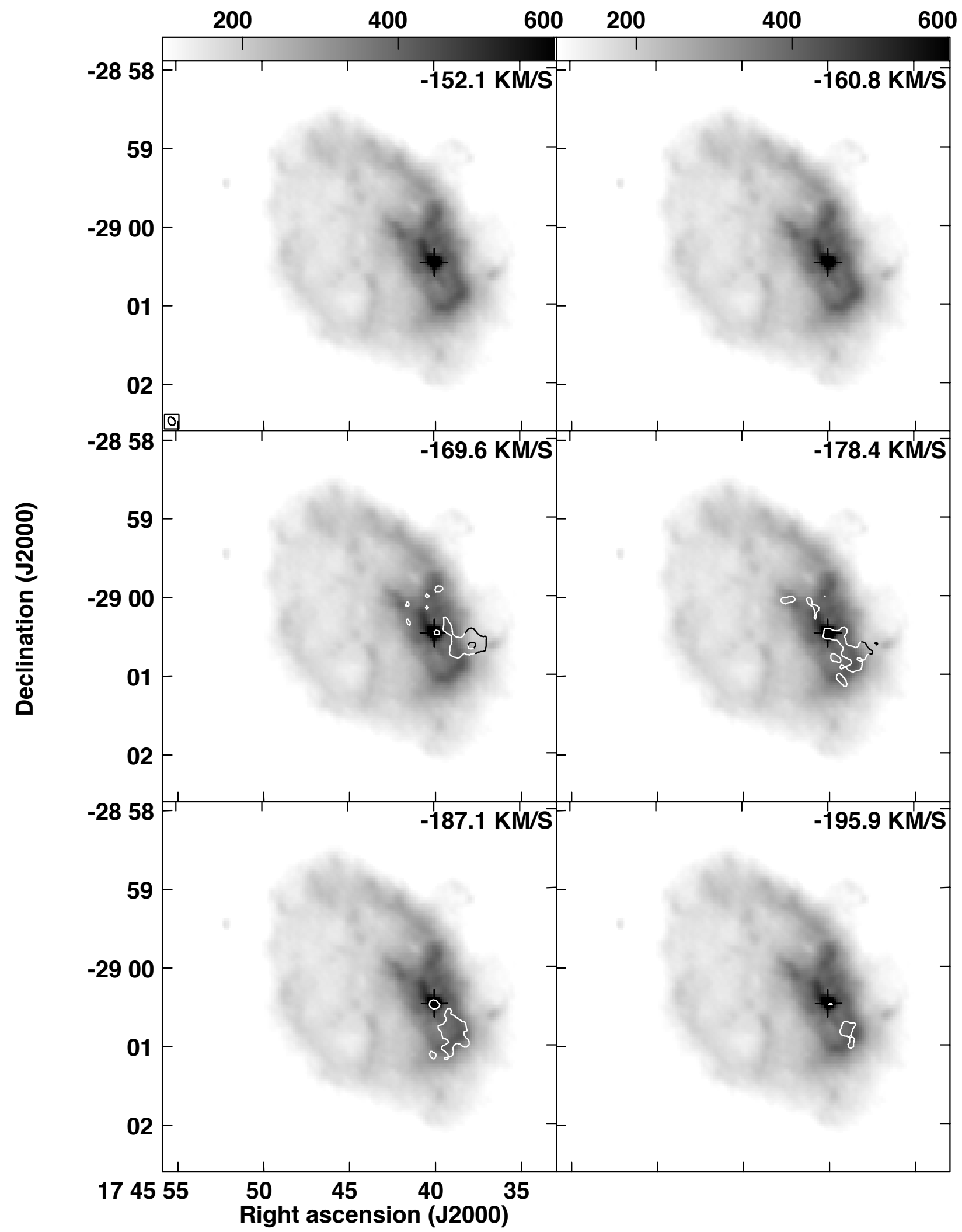

Fig. B.6. 1667-MHz OH-absorption at $-152>V_{\mathrm{LSR}}>-196 \mathrm{~km} \mathrm{~s}^{-1}$. The lowest contour level is $50 \mathrm{mJy} /$ beam $(\sim 2 \sigma)$, and the contour spacing is also $\sim 2 \sigma$. The wedge scale is in $\mathrm{mJy} / \mathrm{beam}$. The position of $\mathrm{Sgr} \mathrm{A}^{*}$ is labelled with a plus sign. 
Appendix C: $-T_{L} / T_{C}$ at $1667 \mathrm{MHz}$

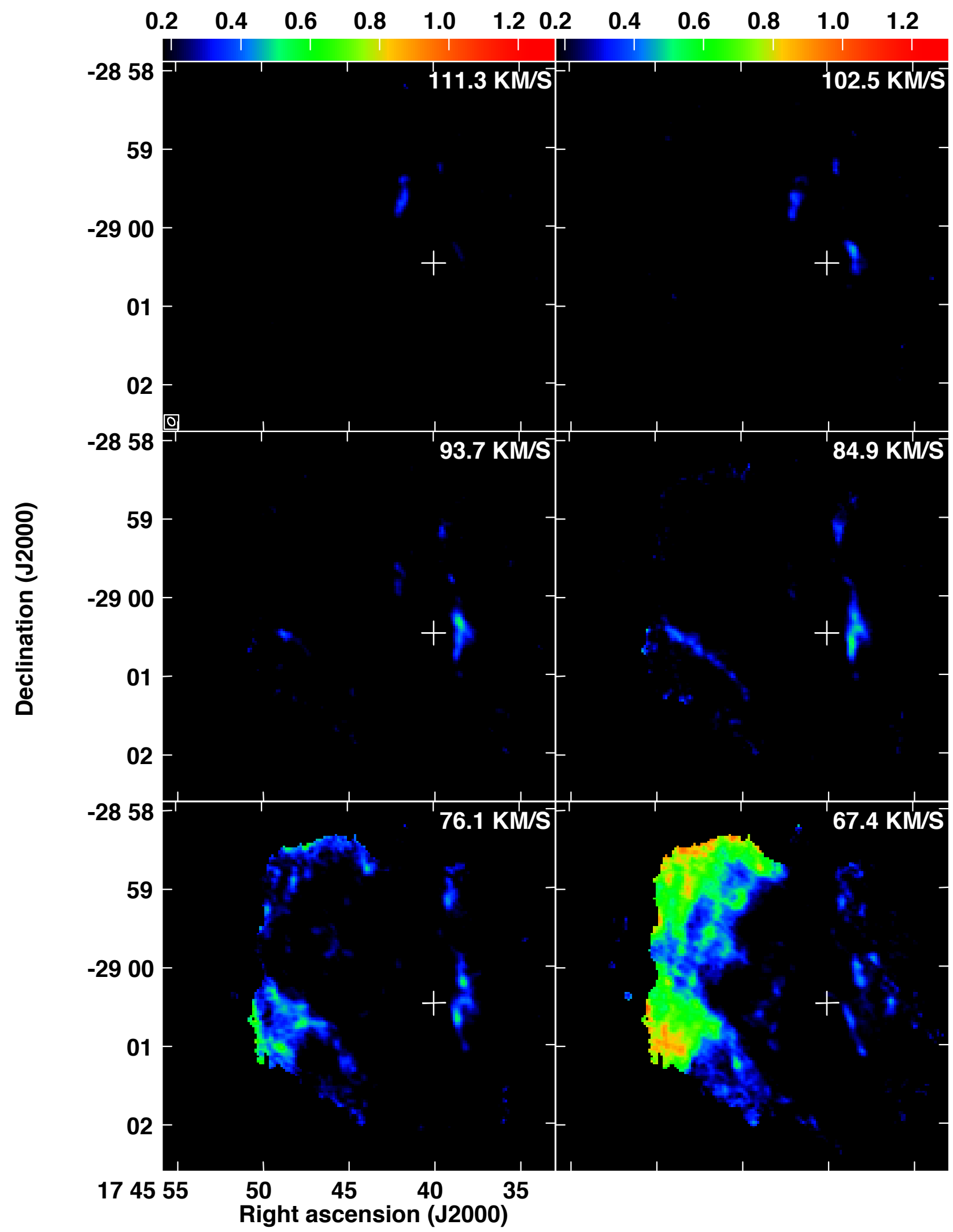

Fig. C.1. $-T_{\mathrm{L}} / T_{\mathrm{C}}$ at $1667 \mathrm{MHz} 111>V_{\mathrm{LSR}}>67 \mathrm{~km} \mathrm{~s}^{-1}$. The position of $\mathrm{Sgr} \mathrm{A} *$ is shown with a plus sign. 
R. Karlsson et al.: $\mathrm{OH}$ in GC molecular clouds

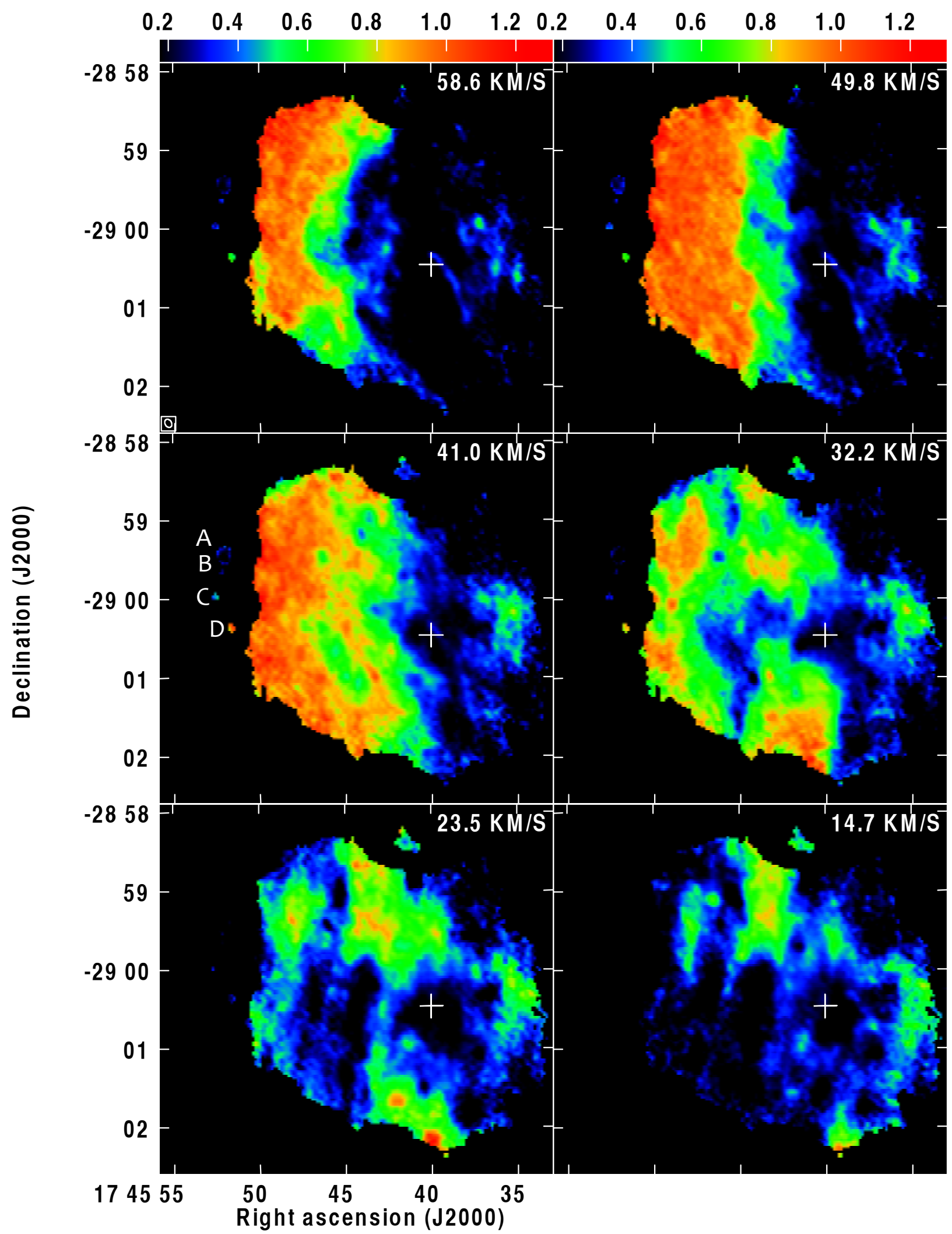

Fig. C.2. $-T_{\mathrm{L}} / T_{\mathrm{C}}$ at $1667 \mathrm{MHz} 59>V_{\mathrm{LSR}}>15 \mathrm{~km} \mathrm{~s}^{-1}$. The position of $\mathrm{Sgr} \mathrm{A}^{*}$ is shown with a plus sign, and the four compact $\mathrm{H}$ II regions are marked by letters A-D. 


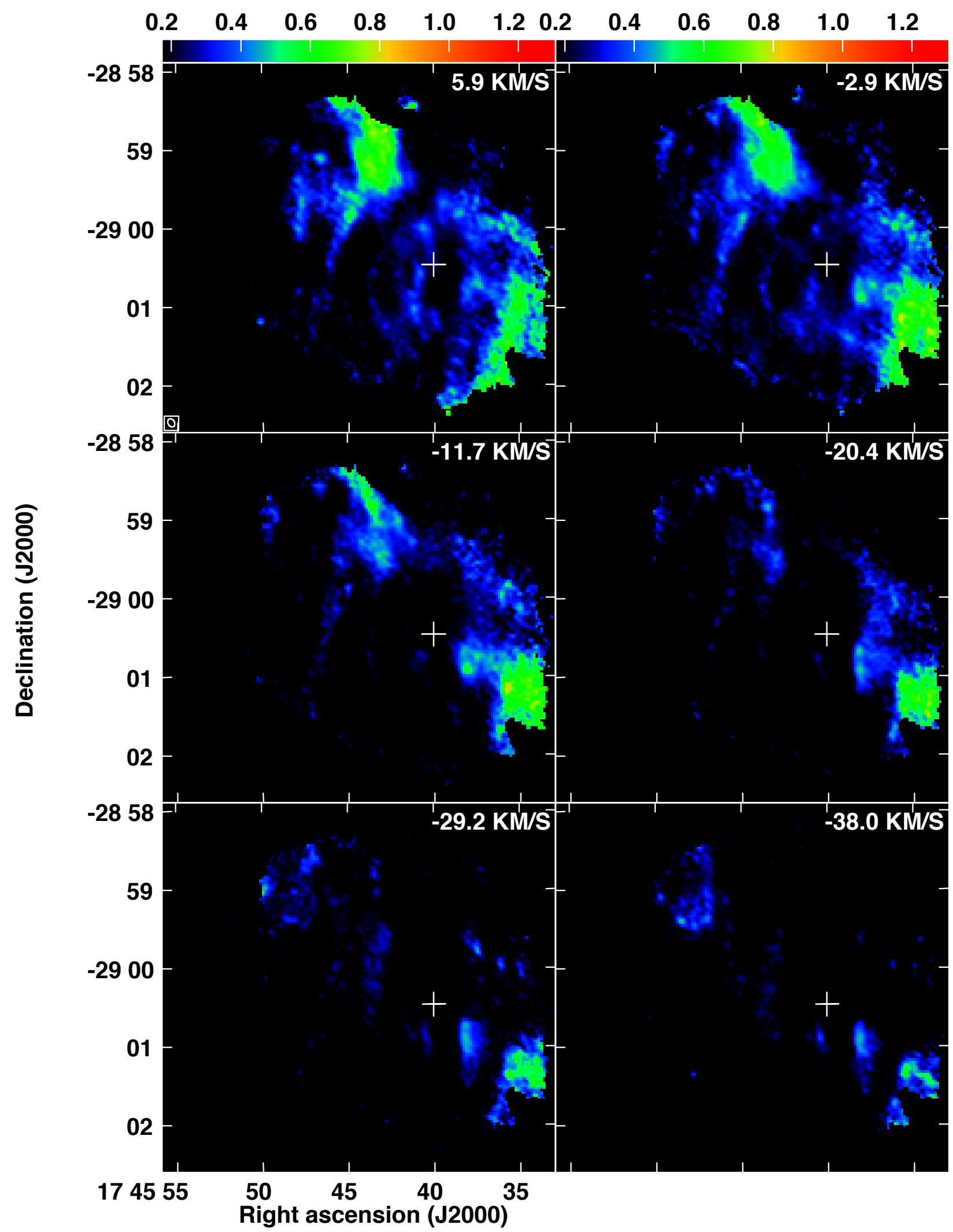

Fig. C.3. $-T_{\mathrm{L}} / T_{\mathrm{C}}$ at $1667 \mathrm{MHz} 6>V_{\mathrm{LSR}}>-38 \mathrm{~km} \mathrm{~s}^{-1}$. The position of $\mathrm{Sgr} \mathrm{A} *$ is shown with a plus sign. 
R. Karlsson et al.: $\mathrm{OH}$ in GC molecular clouds

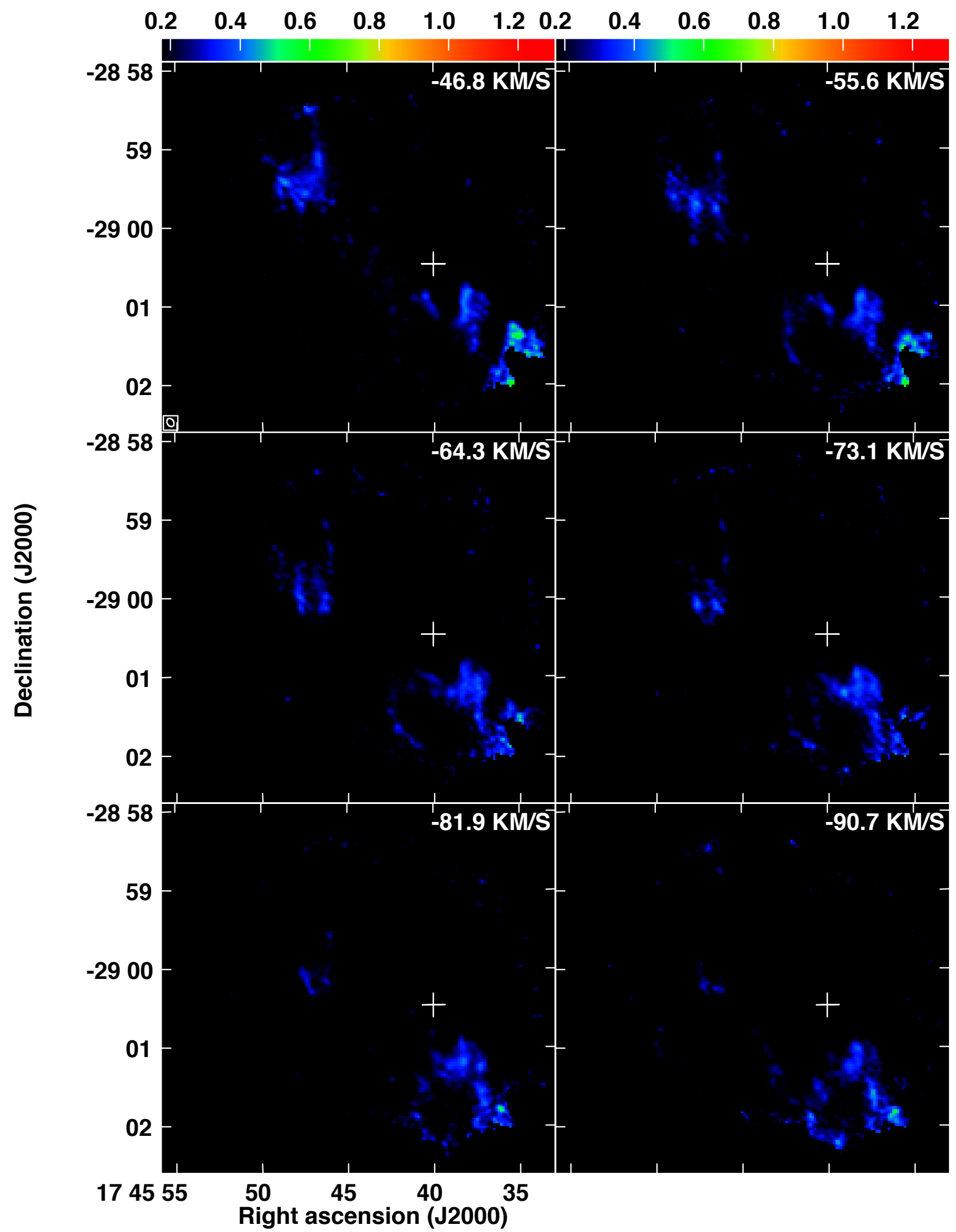

Fig. C.4. $-T_{\mathrm{L}} / T_{\mathrm{C}}$ at $1667 \mathrm{MHz}-47>V_{\mathrm{LSR}}>-91 \mathrm{~km} \mathrm{~s}^{-1}$. The position of $\mathrm{Sgr} \mathrm{A} *$ is shown with a plus sign. 


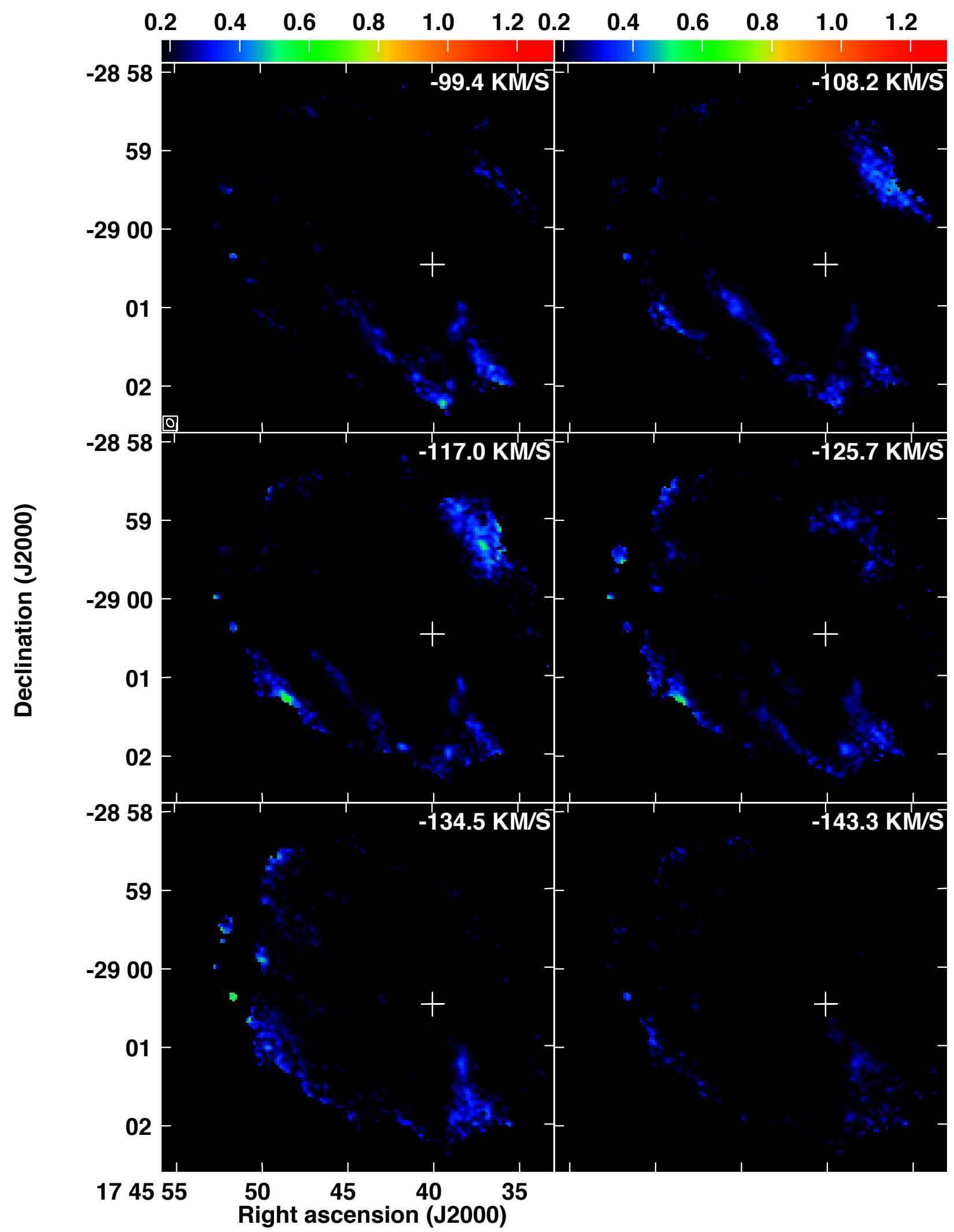

Fig. C.5. $-T_{\mathrm{L}} / T_{\mathrm{C}}$ at $1667 \mathrm{MHz}-99>V_{\mathrm{LSR}}>-143 \mathrm{~km} \mathrm{~s}^{-1}$. The position of $\mathrm{Sgr} \mathrm{A} *$ is shown with a plus sign. 
R. Karlsson et al.: $\mathrm{OH}$ in GC molecular clouds

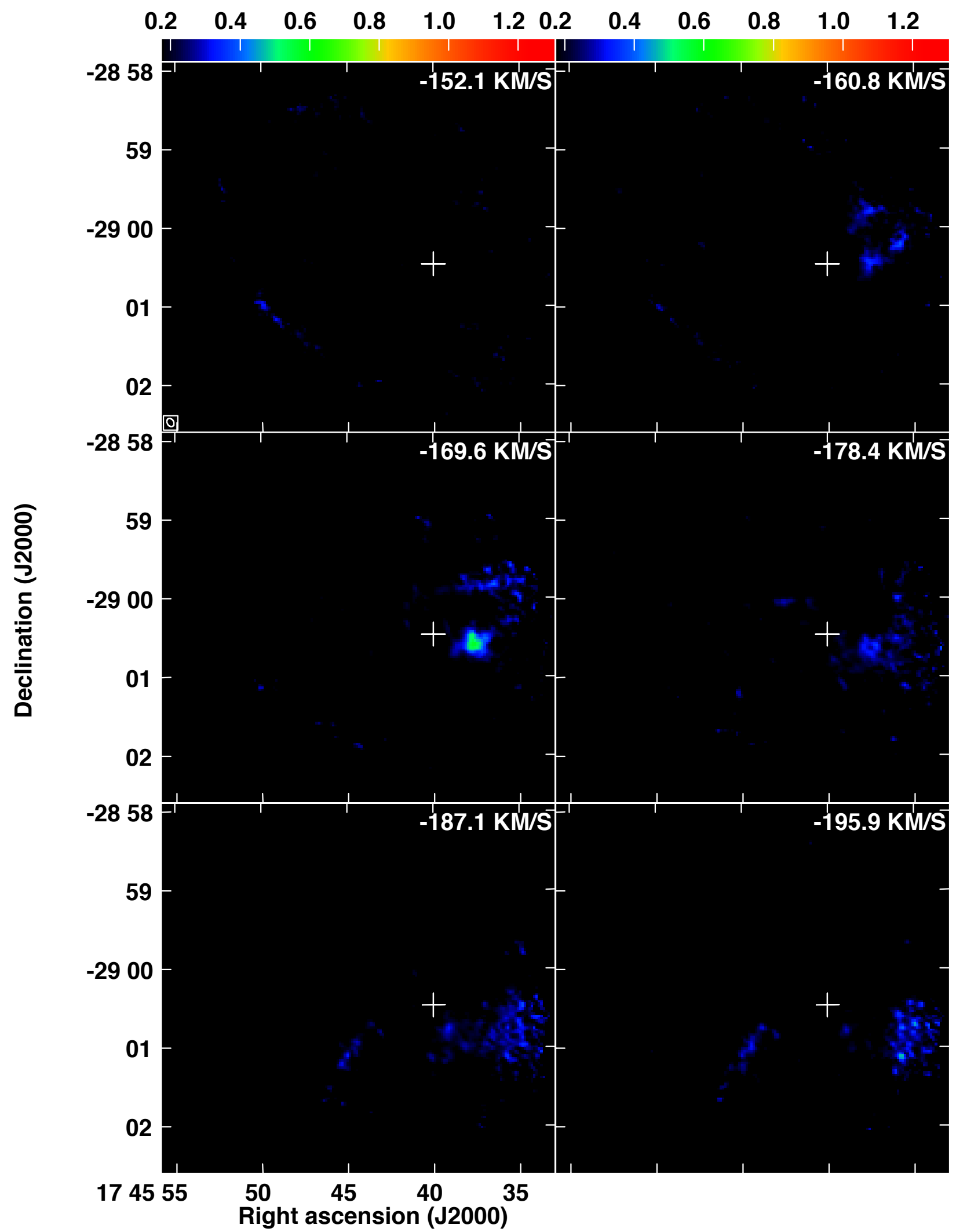

Fig. C.6. $-T_{\mathrm{L}} / T_{\mathrm{C}}$ at $1667 \mathrm{MHz}-152>V_{\mathrm{LSR}}>-196 \mathrm{~km} \mathrm{~s}^{-1}$. The position of $\mathrm{Sgr} \mathrm{A}^{*}$ is shown with a plus sign. 
Appendix D: 1665 MHz OH-absorption

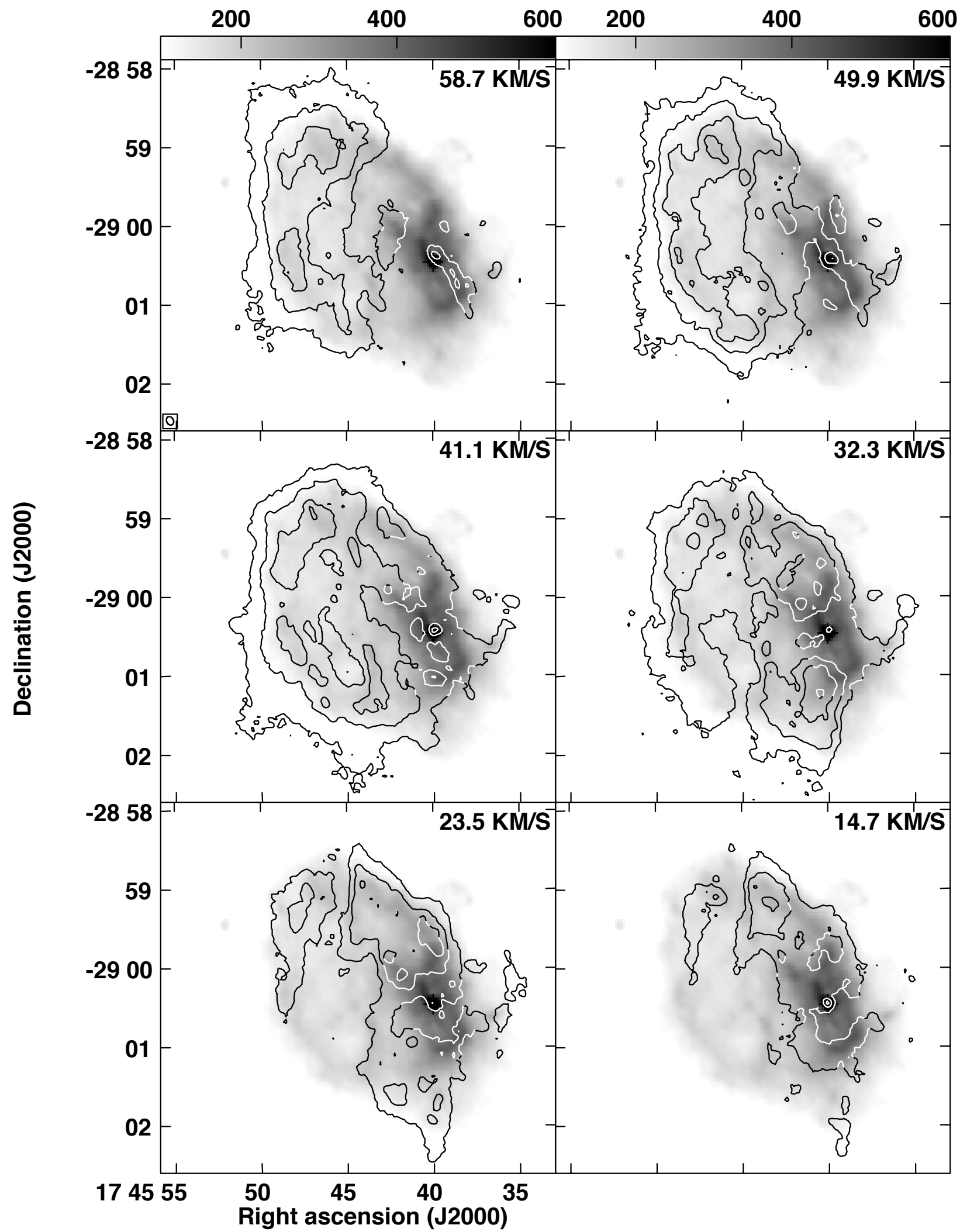

Fig. D.1. 1665-MHz OH-absorption at $59>V_{\mathrm{LSR}}>15 \mathrm{~km} \mathrm{~s}^{-1}$. The lowest contour level is $50 \mathrm{mJy} /$ beam $(\sim 2 \sigma)$, and the contour spacing is also $\sim 2 \sigma$. The wedge scale is in $\mathrm{mJy} /$ beam. The position of Sgr $\mathrm{A} *$ is labelled with a plus sign. 
Appendix $\mathrm{E}:-T_{\mathrm{L}} / T_{\mathrm{C}}$ at $1665 \mathrm{MHz}$

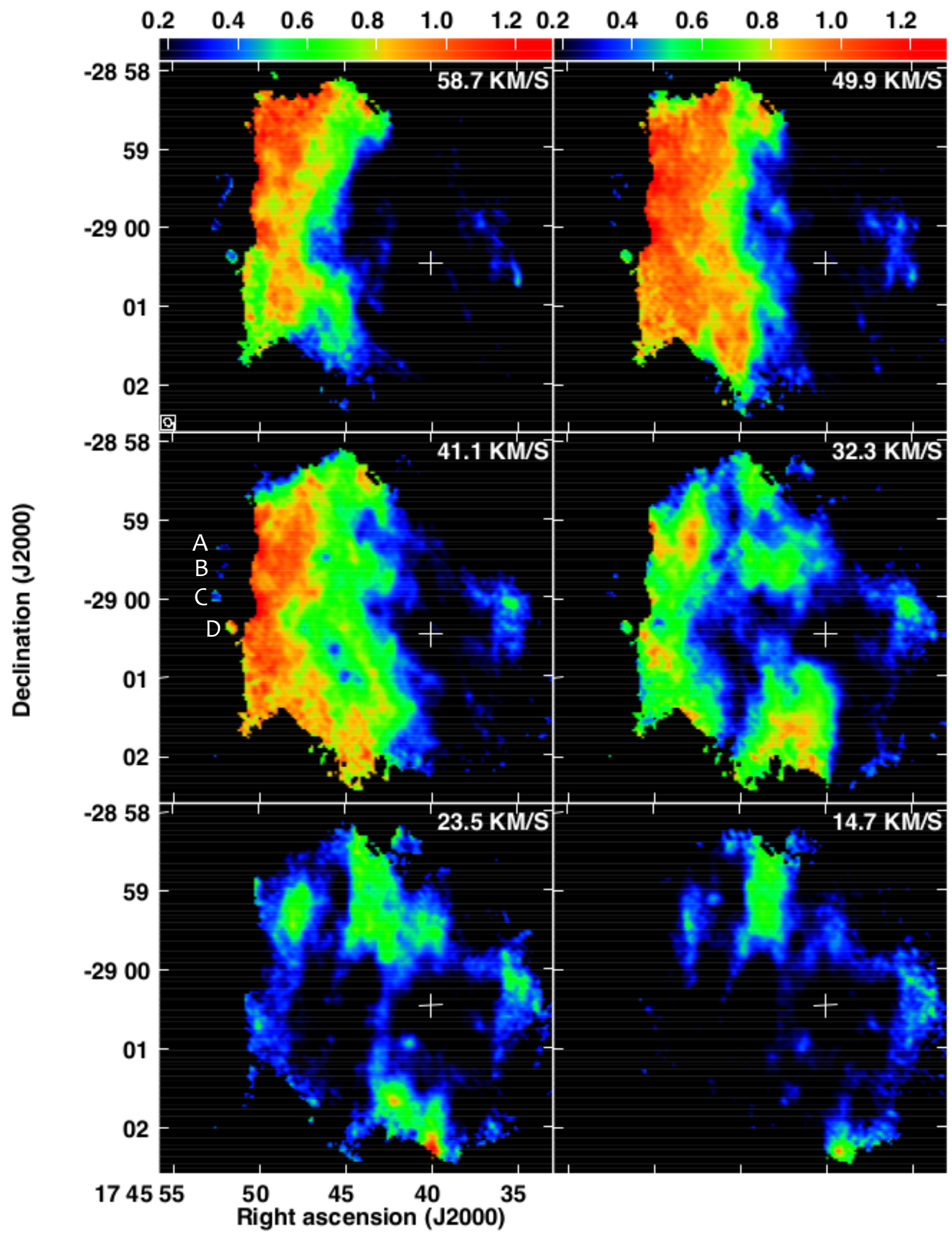

Fig. E.1. $-T_{\mathrm{L}} / T_{\mathrm{C}}$ at $1665 \mathrm{MHz}$ at $59>V_{\mathrm{LSR}}>15 \mathrm{~km} \mathrm{~s}^{-1}$. The position of $\mathrm{Sgr} \mathrm{A} *$ is labelled with a plus sign, and the four compact $\mathrm{H}$ II regions are labelled by letters A-D. 


\section{Appendix $\mathrm{F}: \mathrm{H}_{2}^{18} \mathrm{O}$ in the $\mathrm{OH}-\mathrm{streamer} /+80 \mathrm{~km} \mathrm{~s}^{-1}$ cloud/CND SW shock region}

To further investigate the proposed shock region towards the SW part of the CND, we have used the Odin satellite to observe the water isotope, $\mathrm{H}_{2}^{18} \mathrm{O}$, which is tracer of shock (or strong UV) regions (Karlsson et al. 2013). The observations were performed towards the position (RA, Dec) of $17^{\mathrm{h}} 45^{\mathrm{m}} 39^{\prime} \cdot 7,-29^{\circ} 01^{\prime} 18^{\prime \prime}$ (J2000.0) during April 2013 and April 2014. The total ONsource integration time of the combined data was $62.5 \mathrm{~h}$. An $\mathrm{H}_{2}^{16} \mathrm{O}$ profile was also obtained towards the same position in February 2013, with a total ON-source integration time of $9.4 \mathrm{~h}$. Odin's HPBW at the $\mathrm{H}_{2} \mathrm{O}$ frequencies is 2 ! 1 . The profiles are shown in Fig. F.1, together with $\mathrm{C}^{18} \mathrm{O} J=1-0$ and 2-1 profiles obtained with SEST. We refer to Karlsson et al. (2013) for a detailed description of Odin and SEST observations and analysis of the Sgr A complex region. Here we simply present our new results and their interpretation.

The $\mathrm{H}_{2}^{18} \mathrm{O}$ profile shows a remarkably wide absorption component at positive velocities, in addition to the well-known EMR and 3-kpc Arm features near -130 and $-50 \mathrm{~km} \mathrm{~s}^{-1}$, respectively. This positive-velocity region covers the velocity range of the interacting components discussed in the main part of the paper, viz. the OH-Streamer, the $+80 \mathrm{~km} \mathrm{~s}^{-1}$ cloud and the $+20 \mathrm{~km} \mathrm{~s}^{-1}$ cloud/SS. We obtain a total column density of the order of $N\left(\mathrm{H}_{2}^{18} \mathrm{O}\right) \sim 2.2 \times 10^{14} \mathrm{~cm}^{-2}$, which corresponds to $N\left(\mathrm{H}_{2}^{16} \mathrm{O}\right) \sim 5.5 \times 10^{16}$, assuming a $\mathrm{H}_{2}^{16} \mathrm{O} / \mathrm{H}_{2}^{18} \mathrm{O}$ abundance ratio of 250 in the inner Galactic centre (Wilson $\&$ Rood 1994). From our two $\mathrm{C}^{18} \mathrm{O}$ lines we obtain, in the same region and velocity interval, a molecular hydrogen column density of $N\left(\mathrm{H}_{2}\right) \approx 4.0 \times 10^{22} \mathrm{~cm}^{-2}$, assuming a $\mathrm{C}^{18} \mathrm{O} / \mathrm{H}_{2}$ abundance ratio of $2 \times 10^{-7}$ (Goldsmith 1999). These values result in an abundance ratio $\left[0-\mathrm{H}_{2} \mathrm{O} / \mathrm{H}_{2}\right]=X(\mathrm{o}-$ $\left.\mathrm{H}_{2} \mathrm{O}\right) \sim 1.4 \times 10^{-6}$. Such a high abundance ratio of $\mathrm{H}_{2} \mathrm{O}$ is

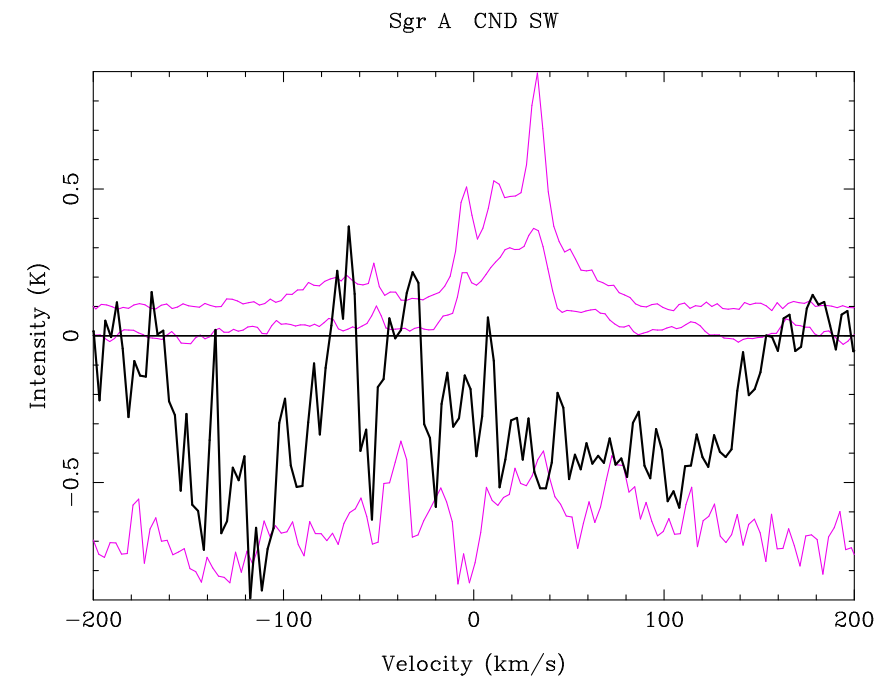

Fig. F.1. $\mathrm{H}_{2}^{18} \mathrm{O}$ (black line), $\mathrm{H}_{2}^{16} \mathrm{O}$ (lower magenta line), $\mathrm{C}^{18} \mathrm{O} J=1-0$ (middle magenta line), and $\mathrm{C}^{18} \mathrm{O} J=2-1$ (upper magenta line) profiles towards the SW position in the CND, coinciding with the position of interaction between the $\mathrm{OH}$ Streamer, the $+80 \mathrm{~km} \mathrm{~s}^{-1}$ cloud and the $\mathrm{SS} /+20 \mathrm{~km} \mathrm{~s}^{-1}$ cloud. The $\mathrm{H}_{2}^{18} \mathrm{O}$ antenna temperature scale has been multiplied by a factor of 10 . The $\mathrm{H}_{2}^{16} \mathrm{O}$ antenna temperature scale has been lowered by $0.7 \mathrm{~K}$ for clarity. The intensity scales of the two $\mathrm{C}^{18} \mathrm{O}$ profiles are in units of brightness temperature, the $J=2-1$ profile having been raised by $0.1 \mathrm{~K}$ for clarity. The channel resolution is $3 \mathrm{~km} \mathrm{~s}^{-1}$ for all the profiles.

comparable to what is found in, for example, the low-velocity outflow region of Orion (Persson et al. 2007), where it has been interpreted as desorption of water ice from dust grains due to shock effects. 